\title{
ON SUPERCONVERGENCE OF A GRADIENT FOR FINITE ELEMENT METHODS FOR AN ELLIPTIC EQUATION WITH THE NONSMOOTH RIGHT-HAND SIDE ${ }^{1}$
}

\author{
ALEXANDER ZLOTNIK \\ Moscow Power Engineering Institute, Department of Mathematical Modelling \\ Krasnokazarmennaya 14, 111250 Moscow, Russia \\ E-mail: zlotnik@apmsun.mpei.ac.ru
}

\begin{abstract}
The elliptic equation under the nonhomogeneous Dirichlet boundary condition in $2 \mathrm{D}$ and $3 \mathrm{D}$ cases is solved. A rectangular nonuniform partition of a domain and polylinear finite elements are taken. For the interpolant of the exact solution $u$, a priori error estimates in the $W^{1,2}$-norm of order $O\left(|h|^{1+\alpha}\right), 0 \leqslant \alpha \leqslant 1$, are proved provided that $u$ possesses a weakened smoothness of order $2+\alpha$ in terms of the Nikolskii or Sobolev spaces. In the case of $\alpha=1$ they involve the third order mixed derivatives of $u$ only. Next error estimates are in terms of data. An estimate of order $O\left(|h|^{1+\alpha}\right), 0<\alpha<1$, is established for the right-hand side $f$ of the equation having a generalized smoothness of order $\alpha$ (which can be the case with respect to all the variables, except one only). In particular, for $\alpha=\frac{1}{2}$ the case of discontinuous $f$ is covered under fairly broad assumptions on the curves (surfaces) of discontinuities. An error estimate of higher order $O\left(\left.|h|^{2}|\log | h\right|^{\frac{1}{2}}\right)$ is proved for the discontinuities lying on the lines (planes) parallel to the coordinate ones only (but situated arbitrarily with respect to the partition). Error estimates of order $O\left(\left.|h|^{2}|\log | h\right|^{\sigma}\right), \sigma=\frac{1}{2}, \frac{3}{2}$, are derived in the case of $f$ which is not compatible with the boundary function; for $u \in W^{3,2}$, this compatibility is necessary. The proofs are based on some propositions from the theory of functions. The corresponding lower error estimates are also included; they justify the sharpness of the estimates without the logarithmic multipliers. Finally, we prove similar results in the case of $2 \mathrm{D}$ linear finite elements and a uniform partition.
\end{abstract}

2000 Mathematics Subject Classification: 65N15; 65N30.

Keywords: elliptic equation, nonsmooth right-hand side, finite element method, gradient superconvergence, lower error estimates.

\section{Introduction}

There exists a vast literature devoted to superconvergence phenomena for the finite element method, in particular see $[8,9,15,19]$ (this list is far from being complete) and references therein.

In this paper, the elliptic equation with variable coefficients is studied under the nonhomogeneous Dirichlet boundary condition in a $2 \mathrm{D}$ or a $3 \mathrm{D}$ rectangular parallelepiped $\Omega$. In

\footnotetext{
${ }^{1}$ This work was partially supported financially by the Russian Basic Research Foundation, grant No. 00-01-00207
} 
Section 2, a rectangular nonuniform partition of $\Omega$ and polylinear finite elements are considered. For the interpolant of the exact solution $u$, we first prove nonstandard a priori error estimates in the $W^{1,2}(\Omega)$-norm of order $O\left(|h|^{1+\alpha}\right), 0 \leqslant \alpha \leqslant 1$, provided that $u$ possesses a weakened smoothness of order $2+\alpha$ in terms of the Nikolskii or Sobolev spaces. For example, in the case of $\alpha=1$ they involve the third order mixed derivatives of $u$ in $L^{2}(\Omega)$ only.

Next, in Section 3 we derive strengthened error estimates in terms of the data which are the main new results of the paper. An error estimate of order $O\left(|h|^{1+\alpha}\right), 0<\alpha<1$, is established for the right-hand side $f$ of the equation having a generalized smoothness of order $\alpha$ in the Nikolskii sense in $L^{2}(\Omega)$; for example, this smoothness can be with respect to any one variable (in the $2 \mathrm{D}$ case) or two variables (in $3 \mathrm{D}$ case) only. In particular, for $\alpha=\frac{1}{2}$ the case of discontinuous $f$ is covered under fairly broad assumptions on the curves (surfaces) of discontinuities. Further, an error estimate of higher order $O\left(|h|^{2}|\log | h||^{\frac{1}{2}}\right)$ is proved in the case of piecewise smooth $f$ having discontinuities on the lines (planes) parallel to the coordinate ones only; the discontinuities can be situated arbitrarily with respect to the partition (for instance, the latter may be uniform). Finally, error estimates of order $O\left(|h|^{2}|\log | h||^{\sigma}\right), \sigma=\frac{1}{2}, \frac{3}{2}$, are derived in the case of $f$ which does not satisfy the restrictive compatibility condition with the boundary function; note that this condition is necessary if one considers the standard case $u \in W^{3,2}(\Omega)$ in order to get the $O\left(|h|^{2}\right)$-error estimate. All the error estimates are based on nontrivial propositions from the theory of functions which are also proved in the section.

In Section 4, we prove the lower error estimates in the simplest case of the generalized Poisson equation under a homogeneous boundary condition. These show that the error estimates (without the logarithmic multipliers) in Section 3 are sharp in order and, moreover, they cannot be strengthened for substantially more restricted Hölder type spaces of $f$.

Error estimates similar to those given in Sections 2 and 3 are also proved in the case of 2D linear finite elements for the uniform partition of $\Omega$ in Section 5. The case of pointwise approximation of the leading coefficients of the equation is also taken into account. The proof of a priori error estimates of order $O\left(|h|^{1+\alpha}\right), 0 \leqslant \alpha \leqslant 1$, differs from that given for polylinear elements.

The appendix is devoted to the proof of a theorem, giving the necessary and sufficient condition for the first derivatives $D_{k} u$ of the solution to belong to $W^{2,2}(\Omega)$. This theorem is essential for deriving error estimates in terms of the data given in Sections 3 and 5.

The main results of Sections 2-4 were announced in [23]; proofs were included in Chapter 2 of the author's D. Sc. thesis [25] which is hardly accessible to the reader.

\section{Nonstandard a priori superconvergence of a gradient}

2.1. We shall solve the elliptic equation

$$
\begin{gathered}
L u \equiv-\sum_{i, j=1}^{n} D_{i}\left(a_{i j} D_{j} u\right)+\sum_{i=1}^{n} b_{i} D_{i} u+a u=f \\
\quad \text { in } \quad \Omega=\left(0, X_{1}\right) \times \cdots \times\left(0, X_{n}\right)
\end{gathered}
$$

under the nonhomogeneous Dirichlet boundary condition

$$
u=g \text { on } \partial \Omega \text {. }
$$


We impose the standard ellipticity condition uniform in $\Omega$ :

$$
\nu \sum_{i=1}^{n} \xi_{i}^{2} \leqslant \sum_{i, j=1}^{n} a_{i j}(x) \xi_{i} \xi_{j}, \quad \forall x=\left(x_{1}, \ldots, x_{n}\right) \in \Omega, \quad \forall \xi_{1}, \ldots, \xi_{n},
$$

where $\nu \geqslant N^{-1}$ and $N>1$ is a parameter. Concerning the coefficients of $L$ we assume that

$$
\left\|a_{i j}\right\|_{W^{1, \infty}(\Omega)}+\left\|b_{i}\right\|_{L^{\infty}(\Omega)}+\|a\|_{L^{\infty}(\Omega)} \leqslant N, \quad \forall i, j .
$$

Let also $\lambda=0$ be not a point of the spectrum of the problem (2.1), (2.2), i.e., for $f=0$ and $g=0$, there exists no solution $u \neq \equiv 0$ from $W^{2,2}(\Omega)$. The latter condition is certainly satisfied provided that

$$
\nu_{0}\|w\|_{H^{\langle 1\rangle}}^{2} \leqslant \mathcal{L}_{\Omega}(w, w), \quad \forall w \in H^{\langle 1\rangle}=W_{0}^{1,2}(\Omega),
$$

where

$$
\mathcal{L}_{\Omega}(w, \varphi) \equiv \int_{\Omega}\left[\sum_{i, j=1}^{n} a_{i j} D_{j} w D_{i} \varphi+\left(\sum_{i=1}^{n} b_{i} D_{i} w+a w\right) \varphi\right] d x
$$

is the bilinear form corresponding to $L$ and $\nu_{0}>0$.

We shall express the conditions on the function $g$ in terms of its extension $g_{\Omega}$ to $\Omega$, which is rather customary [11]. The following inequality holds [11]:

$$
\|u\|_{W^{2,2}(\Omega)} \leqslant c\left(\|f\|_{\Omega}+\left\|g_{\Omega}\right\|_{W^{2,2}(\Omega)}\right),
$$

where $\|\cdot\|_{G}=\|\cdot\|_{L^{2}(G)}$ for any measurable set $G \subset \mathbb{R}^{n}$. In more detail, for $f \in L^{2}(\Omega)$ and $g_{\Omega} \in W^{2,2}(\Omega)$, there exists a unique solution $u \in W^{2,2}(\Omega)$ to the problem (2.1), (2.2) and $u$ satisfies the latter bound. It is essential that in what follows below all the estimates for $u$ and various error estimates are understood similarly for the sake of brevity.

For $0<\alpha<1$, we define the seminorms

$$
\|w\|_{G}^{\left[\alpha \chi_{k}\right]}=\sup _{0<\gamma<X_{k}} \gamma^{-\alpha}\left\|\Delta_{\gamma \chi_{k}} w\right\|_{L^{2}\left(G_{\gamma \chi_{k}}\right)}, \quad\|w\|^{\left[\alpha \chi_{k}\right]}=\|w\|_{\Omega}^{\left[\alpha \chi_{k}\right]}
$$

where $\Delta_{y} w(x)=w(x+y)-w(x)$, the vectors $\chi_{1}, \ldots, \chi_{n}$ form the standard coordinate basis in $\mathbb{R}^{n}$, and $G_{y}=\{x \in G ; x+y \in G\}$. We shall exploit the Nikolskii spaces $H^{\alpha \chi_{k}, 2}(\Omega), H^{\alpha, 2}(\Omega)$ [14] equipped with the norms

$$
\begin{gathered}
\|w\|^{\left(\alpha \chi_{k}\right)}=\|w\|_{H^{\alpha \chi_{k}, 2}(\Omega)} \equiv\|w\|_{\Omega}+\|w\|^{\left[\alpha \chi_{k}\right]} \\
\|w\|^{(\alpha)}=\|w\|_{H^{\alpha, 2}(\Omega)} \equiv\|w\|_{\Omega}+\sum_{i=1}^{n}\|w\|^{\left[\alpha \chi_{i}\right]}
\end{gathered}
$$

and the anisotropic Sobolev space $W^{\chi_{k}, 2}(\Omega)$ equipped with the norm

$$
\|w\|^{\left(\chi_{k}\right)}=\|w\|_{W^{\chi_{k}, 2}(\Omega)} \equiv\|w\|_{\Omega}+\left\|D_{k} w\right\|_{\Omega}, \quad 1 \leqslant k \leqslant n .
$$

Let us extend the definitions of the seminorm and the norm by setting

$$
\|w\|^{\left[0 \chi_{k}\right]}=\|w\|^{(0)}=\|w\|_{\Omega}, \quad\|w\|^{\left[\chi_{k}\right]}=\left\|D_{k} w\right\|_{\Omega}, \quad\|w\|^{(1)}=\|w\|_{W^{1,2}(\Omega)} .
$$

For $1 \leqslant k \leqslant n$ and $0<\alpha<1$, we also introduce the spaces $H_{0}^{\alpha \chi_{k}, 2}(\Omega)$ equipped with the norm

$$
\|w\|_{H_{0}^{\alpha \chi_{k}, 2}(\Omega)}=\|w\|_{\Omega}+\left\|(w)_{\Omega}^{0}\right\|_{\mathbb{R}^{n}}^{\left[\alpha \chi_{k}\right]},
$$


with $(w)_{\Omega}^{0}$ being the extension of $w$ by 0 from $\Omega$ to $\mathbb{R}^{n}$, and the subspaces

$$
W_{0}^{\chi_{k}, 2}(\Omega)=\left\{w \in W^{\chi_{k}, 2}(\Omega) ;\left.w\right|_{\Gamma_{k}}=0\right\} \quad \text { with } \Gamma_{k}=\left\{x \in \partial \Omega ; x_{k}=0, X_{k}\right\}
$$

and $W_{0}^{1,2}(\Omega)=\left\{w \in W^{1,2}(\Omega) ;\left.w\right|_{\partial \Omega}=0\right\}$. We shall need them in Section 3 .

Throughout the paper, for the compatible Banach spaces $B_{0}$ and $B_{1}$, we denote by $\left(B_{0}, B_{1}\right)_{\alpha, \infty}, 0<\alpha<1$, the space constructed by the $K_{\alpha, q^{-}}$method of real interpolation of Banach spaces with $q=\infty[4,17]$. Let in the inequalities given below the constants be independent of the parameter $\alpha$ provided that they are not marked by $\alpha$.

The above-introduced spaces and subspaces are closely connected.

Proposition 2.1. For any $0<\alpha<1$, the following equalities hold:

$$
\begin{aligned}
& \cap_{k} H^{\alpha \chi_{k}, 2}(\Omega)=\left(L^{2}(\Omega), \cap_{k} W^{\alpha \chi_{k}, 2}(\Omega)\right)_{\alpha, \infty}, \\
& \cap_{k} H_{0}^{\alpha \chi_{k}, 2}(\Omega)=\left(L^{2}(\Omega), \cap_{k} W_{0}^{\alpha \chi_{k}, 2}(\Omega)\right)_{\alpha, \infty},
\end{aligned}
$$

with the uniform, with respect to $0<\alpha<1$, equivalence of the norms; here the intersections $\cap_{k}$ are taken over any nonempty subset of indices $\{1, \ldots, n\}$.

In particular,

$$
\begin{gathered}
H^{\alpha, 2}(\Omega)=\left(L^{2}(\Omega), W^{\alpha, 2}(\Omega)\right)_{\alpha, \infty}, \\
H_{0}^{\alpha, 2}(\Omega)=\left(L^{2}(\Omega), W_{0}^{\alpha, 2}(\Omega)\right)_{\alpha, \infty}
\end{gathered}
$$

with the uniform, with respect to $0<\alpha<1$, equivalence of the norms.

Equalities (2.6)-(2.9) follow from Chapters 1,2, and 4 in [17], see also Chapter 6 in [4] or Sections 1.1, 1.2 in [22]; the uniform, with respect to $0<\alpha<1$, equivalence of the norms is clear, in particular, from the latter reference.

For $1 \leqslant k \leqslant n$, we introduce a nonuniform mesh $\bar{\omega}_{k}^{h}$ with the nodes $0=x_{k, 0}<\cdots<$ $x_{k, N_{k}}=X_{k}$ and the steps $h_{k, l}=x_{k, l}-x_{k, l-1}$ with respect to the variable $x_{k}$. Let us set $h_{k}=\max _{1 \leqslant l \leqslant N_{k}} h_{k, l}, h_{k, \min }=\min _{1 \leqslant l \leqslant N_{k}} h_{k, l}$. We define the space $\widehat{S}_{h, k}$ consisting of all the functions continuous on $\left[0, X_{k}\right]$ and linear on all the intervals $\Delta_{k, l-1 / 2}=\left(x_{k, l-1}, x_{k, l}\right), 1 \leqslant l \leqslant N_{k}$, and also set $\widehat{S}_{k}=\left\{\varphi \in \widehat{S}_{h, k} ; \varphi(0)=\varphi\left(X_{k}\right)=0\right\}$. Denote by $s_{k} w$ the function in $\widehat{S}_{h, k}$ coinciding with $w \in C\left[0, X_{k}\right]$ at the nodes of $\bar{\omega}_{k}^{h}$ and, for $w \in L^{1}\left(0, X_{k}\right)$, define the piecewise constant function

$$
[w]_{k}^{h}\left(x_{k}\right)=h_{k, l}^{-1} \int_{x_{k, l-1}}^{x_{k, l}} w(\xi) d \xi \text { for } x_{k} \in \Delta_{k, l-1 / 2}, \quad 1 \leqslant l \leqslant N_{k} .
$$

We introduce the mesh $\bar{\omega}^{h}=\bar{\omega}_{1}^{h} \times \cdots \times \bar{\omega}_{n}^{h}$ in $\bar{\Omega}$ and set $|h|=\max _{1 \leqslant k \leqslant n} h_{k}, h_{\min }=\min _{1 \leqslant k \leqslant n} h_{k, \min }$. Define the spaces

$$
S_{h}=\widehat{S}_{h, 1} \otimes \cdots \otimes \widehat{S}_{h, n}, \quad S^{(1)}=\widehat{S}_{1} \otimes \cdots \otimes \widehat{S}_{n}=\left\{\varphi \in S_{h} ;\left.\varphi\right|_{\partial \Omega}=0\right\} .
$$

The mesh $\bar{\omega}^{h}$ induces the partition $\mathcal{P}_{h}=\{\Pi\}$ of $\bar{\Omega}$ by the rectangular parallelepipeds $\Pi=\Pi_{l_{1}, \cdots, l_{n}}=\bar{\Delta}_{1, l_{1}-1 / 2} \times \cdots \times \bar{\Delta}_{1, l_{n}-1 / 2}$ with the vertices in $\bar{\omega}^{h}$ without common internal points. The space $S_{h}$ consists of all continuous, on $\bar{\Omega}$, functions which are polylinear in 
each parallelepiped $\Pi \in \mathcal{P}_{h}$. We denote by $s w$ the function in $S_{h}$ coinciding with $w \in C(\bar{\Omega})$ at the nodes of $\bar{\omega}^{h}$. Clearly, $s=s_{1} \ldots s_{n}$. We also set

$$
s_{\langle i\rangle}=\prod_{\substack{1 \leqslant k \leqslant n, k \neq i}} s_{k}, \quad s_{\langle i j\rangle}=\prod_{\substack{1 \leqslant k \leqslant n, k \neq i, j}} s_{k} \text { for } j \neq i .
$$

Let $\partial \omega^{h}=\bar{\omega}^{h} \cap \partial \Omega$. The mesh $\bar{\omega}^{h}$ is said to be uniform provided that the meshes $\bar{\omega}_{k}^{h}$ are uniform for all $k$.

2.2. To solve the problem (2.1), (2.2), we first consider the well-known finite element method such that an approximate solution $v \in S_{h}$ satisfies the integral identity

$$
\mathcal{L}_{\Omega}(v, \varphi)=\int_{\Omega} f \varphi d x, \quad \forall \varphi \in S^{(1)}
$$

and the mesh boundary condition $v=g$ on $\partial \omega^{h}$.

In what follows, we need the inequality

$$
\|w\|_{H^{\langle 1\rangle}} \leqslant c_{1} \sup _{\varphi \in S^{(1)}} \frac{\mathcal{L}_{\Omega}(w, \varphi)}{\|\varphi\|_{H^{\langle 1\rangle}}}, \quad \forall w \in S^{(1)}
$$

This certainly follows from inequality $(2.4)$, with $c_{1}=\frac{1}{\nu_{0}}$. In the general case inequality (2.10) is valid at least for all sufficiently small $|h| \leqslant h_{0}$; this can be shown with the aid of the method described in Section 4.2 in [15]. Hereafter, the constants $c, c_{1}, c_{2}, \ldots$ are independent of the mesh. By virtue of (2.10), the approximate solution exists and is unique for $f \in L^{2}(\Omega)$ and $g \in C(\partial \Omega)$.

Let us establish the estimates for $\|s u-v\|_{H^{\langle 1\rangle}}$, which express the superconvergence of the gradient [8] for the method under consideration both in $2 \mathrm{D}$ and 3D cases (i.e., for $n=2,3)$. Let $f \in L^{2}(\Omega), g_{\Omega} \in W^{2,2}(\Omega)$, so that $u \in W^{2,2}(\Omega)$. First, we shall suppose that the second derivatives of $u$ a priori have additional smoothness, which will be explicitly exhibited in the estimates; below in Section 3, estimates in terms of data will be proved. We set $\operatorname{div} \mathbf{b}=\sum_{i=1}^{n} D_{i} b_{i}$ and $\gamma_{2}=1, \gamma_{3}=\frac{|h|}{h_{\min }}$.

Theorem 2.1. Let $\|\operatorname{div} \mathbf{b}\|_{L^{\infty}(\Omega)} \leqslant N$ and $0 \leqslant \alpha \leqslant 1$. The following error estimates, for $n=2$,

$$
\|s u-v\|_{H^{\langle 1\rangle}} \leqslant c \sum_{i=1,2} h_{i}^{1+\alpha}\left(\left\|D_{i}^{2} u\right\|_{\Omega}+\left\|D_{1} D_{2} u\right\|^{\left[\alpha \chi_{i}\right]}\right)
$$

and, for $n=3$,

$$
\begin{aligned}
& \|s u-v\|_{H^{\langle 1\rangle}} \leqslant c \sum_{i=1}^{3} h_{i}^{2}\left(\left\|D_{i}^{2} u\right\|_{\Omega}+\sum_{\substack{1 \leqslant j \leqslant 3 \\
j \neq i}}\left\|D_{i}^{2} D_{j} u\right\|_{\Omega}\right), \\
& \|s u-v\|_{H^{\langle 1\rangle}} \leqslant c \gamma_{3}^{1-\alpha}|h|^{1+\alpha} \sum_{i=1}^{3}\left\|D_{i}^{2} u\right\|^{(\alpha)}
\end{aligned}
$$

are valid. 
Proof. Let $\varphi \in S^{(1)}$. According to the definitions of $u$ and $v$ the error $r=s u-v \in H^{\langle 1\rangle}$ satisfies the identity

$$
\mathcal{L}_{\Omega}(r, \varphi)=-\mathcal{L}_{\Omega}(u-s u, \varphi) .
$$

We set $\rho_{j}=s_{\langle j\rangle} u-s u$ and decompose the right-hand bilinear form:

$$
\begin{aligned}
\mathcal{L}_{\Omega}(u-s u, \varphi)= & \sum_{i, j=1}^{n}\left(\int_{\Omega} a_{i j} D_{j}\left(u-s_{\langle j\rangle} u\right) D_{i} \varphi d x+\int_{\Omega} a_{i j} D_{j} \rho_{j} D_{i} \varphi d x\right) \\
& +\int_{\Omega}\left\{-\sum_{i=1}^{n} b_{i}(u-s u) D_{i} \varphi+(a-\operatorname{divb})(u-s u) \varphi\right\} d x .
\end{aligned}
$$

Let us transform the summands with $D_{j} \rho_{j}$ on the right-hand side as follows. For $i=j$, we represent the integral over $x_{j} \in\left(0, X_{j}\right)$ as a sum of integrals over $x_{j} \in \Delta_{j, l-1 / 2}, 1 \leqslant l \leqslant N_{j}$. By transferring $D_{j}$ from $\rho_{j}$ to the cofactor, using the constancy of $D_{j} \varphi$ over $x_{j} \in \Delta_{j, l-1 / 2}$ and the property $\left.\rho_{j}\right|_{x_{j}=x_{j, l}}=0,0 \leqslant l \leqslant N_{j}$, we get

$$
\int_{\Omega} a_{j j} D_{j} \rho_{j} D_{j} \varphi d x=-\int_{\Omega} D_{j} a_{j j} \cdot \rho_{j} D_{j} \varphi d x
$$

For $i \neq j$, we apply the formula

$$
a_{i j} D_{j} \rho_{j}=D_{j}\left(a_{i j} \rho_{j}\right)-D_{j} a_{i j} \cdot \rho_{j}
$$

In the integrals $\int_{\Omega} D_{j}\left(a_{i j} \rho_{j}\right) D_{i} \varphi d x$, we transfer first $D_{j}$ and next $D_{i}$ to the cofactors. The substitutions equal zero by virtue of the condition $\left.\varphi\right|_{\partial \Omega}=0$. Therefore

$$
\int_{\Omega} a_{i j} D_{j} \rho_{j} D_{i} \varphi d x=\int_{\Omega}\left\{\left[a_{i j} D_{i} \rho_{j}+D_{i} a_{i j} \cdot \rho_{j}\right] D_{j} \varphi-D_{j} a_{i j} \cdot \rho_{j} D_{i} \varphi\right\} d x .
$$

Now, it follows from (2.15) along with the relations

$$
D_{k} \rho_{j}=\left[D_{k}\left(s_{\langle k j\rangle} u-s_{\langle k\rangle} u\right)\right]_{k}^{h} \text { for } k \neq j, \quad\left\|[w]_{k}^{h}\right\|_{\left(0, X_{k}\right)} \leqslant\|w\|_{\left(0, X_{k}\right)}
$$

that the following estimate holds:

$$
\begin{aligned}
\mathcal{L}_{\Omega}(u-s u, \varphi) \leqslant & c\left\{\sum_{i=1}^{n}\left(\left\|D_{i}\left(u-s_{\langle i\rangle} u\right)\right\|_{\Omega}+\sum_{\substack{1 \leqslant j \leqslant n, j \neq i}}\left\|D_{i} \rho_{j}\right\|_{\Omega}+\left\|\rho_{i}\right\|_{\Omega}\right)\right. \\
& \left.+\|u-s u\|_{\Omega}\right\}\|\varphi\|_{H^{\langle 1\rangle}} \\
\leqslant & c^{\prime}\left\{\sum _ { i = 1 } ^ { n } \left(\left\|D_{i}\left(u-s_{\langle i\rangle} u\right)\right\|_{\Omega}+\sum_{\substack{1 \leqslant j \leqslant n, j \neq i}}\left\|D_{i}\left(u-s_{\langle i j\rangle} u\right)\right\|_{\Omega}\right.\right. \\
& \left.\left.+\left\|u-s_{\langle i\rangle} u\right\|_{\Omega}\right)+\|u-s u\|_{\Omega}\right\}\|\varphi\|_{H^{\langle 1\rangle}} .
\end{aligned}
$$


Clearly, the estimate $\mathcal{L}_{\Omega}(u-s u, \varphi) \leqslant c\|u-s u\|^{(1)}\|\varphi\|_{H^{\langle 1\rangle}}$ is valid as well.

According to identity (2.14) and inequality (2.10) we have

$$
\begin{aligned}
& \|r\|_{H^{\langle 1\rangle}} \leqslant c\left\{\sum_{i=1}^{n}\left(\|u-s\langle i\rangle\|^{\left(\chi_{i}\right)}+\sum_{\substack{1 \leqslant j \leqslant n, j \neq i}}\left\|D_{i}\left(u-s_{\langle i j\rangle} u\right)\right\|_{\Omega}\right)+\|u-s u\|_{\Omega}\right\}, \\
& \|r\|_{H^{\langle 1\rangle}} \leqslant c\|u-s u\|^{(1)} .
\end{aligned}
$$

For $i \neq j$, obviously $s_{\langle i j\rangle} u=u$, for $n=2$, or $s_{\langle i j\rangle} u=s_{k} u$ with $(i, j, k)$ being a permutation of indexes $(1,2,3)$, for $n=3$.

For any function $w \in W^{2,2}(\Omega)$, in the cases of $n=1,2,3$, the following approximation error estimate holds:

$$
\|w-s w\|_{\Omega}+\sum_{i=1}^{n} h_{i, \min }\left\|D_{i}(w-s w)\right\|_{\Omega} \leqslant c \sum_{j=1}^{n} h_{j}^{2}\left\|D_{j}^{2} w\right\|_{\Omega}
$$

(which follows from at least the theorem on equivalent norms in $W^{2,2}(\Omega)$, for an example see Section 1.8, Theorem 5 in [15] or Exercise 3.1.1 in [6]). The following estimates hold as well:

$$
\begin{array}{cc}
\left\|w-s_{\langle i\rangle} w\right\|_{\Omega} \leqslant c \sum_{\substack{1 \leqslant j \leqslant n, j \neq i}} h_{j}^{2}\left\|D_{j}^{2} w\right\|_{\Omega}, \quad n=3, & 1 \leqslant i \leqslant n, \\
\left\|w-s_{k} w\right\|_{\Omega} \leqslant c h_{k}^{1+\alpha}\left\|D_{k} w\right\|^{\left[(1+\alpha) \chi_{k}\right]}, & 0 \leqslant \alpha \leqslant 1
\end{array}
$$

(where the condition $w \in L^{2}(\Omega)$ is supposed). Estimate (2.20) follows easily from the $2 \mathrm{D}$ case of estimate (2.19). Since

$$
\left(w-s_{k} w\right)(x)=0 \text { provided that } x_{k} \in \bar{\omega}_{k}^{h}, \quad D_{k}\left(w-s_{k} w\right)=D_{k} w-\left[D_{k} w\right]_{k}^{h},
$$

we have $\left\|w-s_{k} w\right\|_{\Omega} \leqslant h_{k}\left\|D_{k} w-\left[D_{k} w\right]_{k}^{h}\right\|_{\Omega}$. Therefore, by taking into account the well-known properties of $[\cdot]_{k}^{h}$, we first get the known estimate (2.21), for $\alpha=0,1$, next, by exploiting the technique from Lemma 2 in [20] (see also Section 2.2, Lemma 2.2 in [22]), we get this estimate for $0<\alpha<1$ as well.

Error estimates (2.11), for $0 \leqslant \alpha \leqslant 1$, and (2.12) follow from (2.17). To demonstrate this, it suffices to interchange the operators of differentiation and interpolation (here this action is possible) and to apply estimates (2.19)-(2.21). Error estimate (2.13), for $\alpha=0$ and $\alpha=1$, follows from (2.18), (2.19) and (2.12), respectively.

To establish estimate (2.13), for $0<\alpha<1$, we exploit interpolation of linear operators. Here, it is convenient to consider $s u-v=Q u$, where $Q: W^{2,2}(\Omega) \rightarrow H^{\langle 1\rangle}$ is a linear operator; this is possible due to the linear dependence of $v$ on the pair $\{f, g\}=\left\{L u,\left.u\right|_{\partial \Omega}\right\}$. By starting from estimate (2.13), for $\alpha=0,1$, as well as according to the proof of the interpolation theorem for linear operators, see Section 1.3.2 in [4], and with the aid of the embedding of $H^{\langle 1\rangle}$ in $L^{2}(\Omega)$, we get, for $0 \leqslant \alpha \leqslant 1$,

$$
\|Q u\|_{H^{\langle 1\rangle}} \leqslant c M_{\alpha} \sup _{0<\delta \leqslant \delta_{0}}\left[\delta^{-\alpha} \inf _{\psi} \sum_{i=1}^{n}\left(\left\|D_{i}^{2}(u-\psi)\right\|_{\Omega}+\delta\left\|D_{i}^{2} \psi\right\|^{(1)}\right)\right],
$$

where $M_{\alpha}=\gamma_{3}^{1-\alpha}|h|^{1+\alpha}$ and inf is taken over all $\psi \in L^{2}(\Omega)$ having $D_{i}^{2} \psi \in W^{1,2}(\Omega), \quad 1 \leqslant$ $i \leqslant n$. Let $\widetilde{u}$ be an extension of $u$ from $\Omega$ to $\widetilde{\Omega}=\left(-\delta_{0}, X_{1}+\delta_{0}\right) \times \cdots \times\left(-\delta_{0}, X_{n}+\delta_{0}\right)$. By 
choosing a mollification (with the radius $\delta_{0}$ ) of $\widetilde{u}$ as $\psi$, we get, see Section 6.10 in [14] and Section 1.2 in [22],

$$
\|s u-v\|_{H^{\langle 1\rangle}} \leqslant c_{1} M_{\alpha} \sum_{i=1}^{n}\left\|D_{i}^{2} \widetilde{u}\right\|_{\widetilde{\Omega}}^{(\alpha)}, \quad 0 \leqslant \alpha \leqslant 1,
$$

where the subscript $\widetilde{\Omega}$ means that the norm is taken over $\widetilde{\Omega}$ instead of $\Omega$. If, to construct $\widetilde{u}$, one applies the usual Hestenes' extension method (see Section 2.9.1, Lemma 1 in [17] or Section 1.3.1 in [15]) with respect to $x_{1}, \ldots, x_{n}$ sequentially, then $D_{k}^{2} \widetilde{u}=T_{k} D_{k}^{2} u$, where the linear operator $T_{k}$ possesses the property $\left\|T_{k} w\right\|_{\widetilde{\Omega}}^{(\alpha)} \leqslant c\|w\|^{(\alpha)}$, for $\alpha=0,1$ and any $1 \leqslant k \leqslant n$. By virtue of equality (2.8) this property of $T_{k}$ is valid for $0<\alpha<1$ as well. Now estimate (2.13) follows from (2.22).

Notice that in the sequel estimates (2.11) and (2.13), for $0<\alpha<1$, are used in the case of $g \not \equiv 0$, only though sometimes they are useful in the case of $g=0$ as well, see [24].

2.3. Let us return to the boundary-value problem (2.1), (2.2). We impose additional conditions on the coefficients of $L$, namely $a_{i j}=0$ for all $i \neq j$ and

$$
\left\|a_{k k}\right\|_{W^{2, \infty}(\Omega)}+\left\|D_{k} a_{k k}\right\|_{W^{2, \infty}(\Omega)}+\left\|b_{k}\right\|_{W^{1, \infty}(\Omega)}+\|a\|_{W^{1, \infty}(\Omega)} \leqslant N, \quad 1 \leqslant k \leqslant n .
$$

The next theorem establishes the necessary and sufficient condition for the property $D_{k} u \in W^{2,2}(\Omega)$ to be valid. Let $W^{1 / 2,2}(\partial \Omega)$ be the well-known Sobolev-Slobodetskii space, see $[5,15]$, and $H^{\langle 2\rangle}$ be the subspace $\left\{w \in W^{2,2}(\Omega) ;\left.w\right|_{\partial \Omega}=0\right\}$.

Theorem 2.2. Let $g=0$. The following inequalities hold, for $1 \leqslant k \leqslant n$ :

$$
\begin{aligned}
\|u\|_{H^{\langle 2\rangle}}+\left\|D_{k} u\right\|_{W^{2,2}(\Omega)} & \leqslant c_{1}\left(\|f\|_{W^{\chi_{k}, 2}(\Omega)}+\left\|(f)_{\Gamma_{k}}^{0}\right\|_{W^{1 / 2,2}(\partial \Omega)}\right) \\
\|f\|_{W^{\chi_{k}, 2}(\Omega)}+\left\|(f)_{\Gamma_{k}}^{0}\right\|_{W^{1 / 2,2}(\partial \Omega)} & \leqslant c_{2}\left(\|u\|_{H^{\langle 2\rangle}}+\left\|D_{k} u\right\|_{W^{2,2}(\Omega)}\right)
\end{aligned}
$$

with $(f)_{\Gamma_{k}}^{0}$ being the extension of $f$ from $\Gamma_{k}$ to $\partial \Omega \backslash \Gamma_{k}$ by 0 .

The proof of the theorem is given in Appendix.

\section{Superconvergence of a gradient in classes of nonsmooth data}

3.1. Suppose that the coefficients of $L$ satisfy the conditions from Sections 2.1 and 2.3. By starting from Theorems 2.1 and 2.2, we establish the superconvergence of a gradient in the classes of data.

We set $\Omega=(0,1)^{n}$ with no loss of generality. Introduce the function spaces

$$
V^{0}=L^{2}(\Omega), \quad V^{1}=\mathfrak{S} W_{0}^{\chi_{k}, 2}(\Omega), \quad V^{\alpha}=\left(V^{0}, V^{1}\right)_{\alpha, \infty}, 0<\alpha<1 .
$$

Hereafter, for the compatible Banach spaces $B_{1}, \ldots, B_{n}$, we exploit the notation

$$
\mathfrak{S} B_{k}= \begin{cases}B_{1}+B_{2} & \text { for } n=2, \\ B_{1} \cap B_{2}+B_{2} \cap B_{3}+B_{1} \cap B_{3} & \text { for } n=3 .\end{cases}
$$

The sum and intersection of the Banach spaces are understood in the standard sense, see Section 2.3 in [4]. Obviously, the sum of the spaces is broader than any summand (provided that they are pairwise different). 
Theorem 3.1. For $n=2,3$, the following error estimate holds:

$$
\|s u-v\|_{H^{\langle 1\rangle}} \leqslant c \gamma_{n}^{1-\alpha}|h|^{1+\alpha}\left(\left\|f-L g_{\Omega}\right\|_{V^{\alpha}}+\sum_{i=1}^{n}\left\|D_{i}^{2} g_{\Omega}\right\|^{(\alpha)}\right), \quad 0 \leqslant \alpha \leqslant 1,
$$

where the condition $g_{\Omega} \in W^{2,2}(\Omega)$ is supposed.

Proof. We write down the error in the form $r=Q u=Q\left(u-g_{\Omega}\right)+Q g_{\Omega}$, where $r$ and $Q$ were introduced in the proof of Theorem 2.1. To bound $Q g_{\Omega}$, it suffices to apply coarsened estimate (2.11) and estimate (2.13).

Now, we bound $Q\left(u-g_{\Omega}\right)$, i.e., it suffices to prove the following estimate, for $g=0$ :

$$
\|r\|_{H^{\langle 1\rangle}} \leqslant c \gamma_{n}^{1-\alpha}|h|^{1+\alpha}\|f\|_{V^{\alpha}}
$$

where $0 \leqslant \alpha \leqslant 1$. For $\alpha=0$, this estimate follows from bounds (2.11) and (2.13) (both for $\alpha=0$ ) along with inequality (2.5). For $\alpha=1$, to derive (3.2) we first apply (2.11), for $\alpha=1$, and (2.12) and next exploit the fact that the norms in $L^{2}(\Omega)$ of all the third order derivatives of $u$ (excluding nonmixed ones) is possible to bound by the norm of $f$ in any space $W_{0}^{\chi_{k}, 2}(\Omega)$, for $n=2$, or $W_{0}^{\chi_{i}, 2}(\Omega) \cap W_{0}^{\chi_{j}, 2}(\Omega), i \neq j$, for $n=3$. The case $0<\alpha<1$ in (3.2) can be reduced to the cases $\alpha=0,1$ simply by the interpolation theorem for linear operators. Here, it is already convenient to consider $r$ as $r=A f$, where $A: L^{2}(\Omega) \rightarrow H^{\langle 1\rangle}$ is a linear operator.

3.2. The aim of the rest of the section is to derive from estimate (3.1) some important consequences under explicit conditions on $f$ and $g_{\Omega}$. To this end, we need a chain of propositions from the theory of functions.

For $f \in L^{2}(\Omega)$, we introduce the Fourier series expansion with respect to sines

$$
f(x)=\sum_{m>0} \widetilde{f}_{m} \sigma_{m}(x), \quad \tilde{f}_{m}=\int_{\Omega} f \sigma_{m} d x, \quad \sigma_{m}=2^{n / 2} \sin \pi m_{1} x_{1} \ldots \sin \pi m_{n} x_{n},
$$

where $m=\left(m_{1}, \ldots, m_{n}\right)$ is a multiindex and $m>0$ means that $m_{1}>0, \ldots, m_{n}>0$. We set

$$
\langle m\rangle=\left\{\begin{array}{cl}
\min \left\{m_{1}, m_{2}\right\} & \text { for } n=2, \\
\min _{1 \leqslant i<j \leqslant n} m_{i}+m_{j} & \text { for } n=3 .
\end{array}\right.
$$

For $n=3$, by choosing the permutation $(i, j, k)$ of $(1,2,3)$ such that $m_{i} \leqslant m_{j} \leqslant m_{k}$, we have $m_{j} \leqslant\langle m\rangle \leqslant 2 m_{j}$. Let us introduce spaces of functions on $\Omega$ with the norms, for $\alpha \geqslant 0$ and $\chi=(1, \ldots, 1) \in \mathbb{R}^{n}$,

$$
\|f\|_{\mathcal{H}^{\alpha}}=\left(\sum_{m>0}\langle m\rangle^{2 \alpha} \widetilde{f}_{m}^{2}\right)^{1 / 2}, \quad\|f\|_{S_{1}^{\chi} \mathcal{H}}=\sup _{m>0} m_{1} \ldots m_{n}\left|\widetilde{f}_{m}\right| .
$$

Proposition 3.1. 1. The spaces $V^{1}$ and $\mathcal{H}^{1}$ coincide (up to the equivalence of the norms).

2. The following inequality holds:

$$
\|f\|_{V^{\alpha}} \leqslant c\|f\|_{\mathcal{H}^{\alpha}}, \quad 0 \leqslant \alpha \leqslant 1 .
$$


Proof. 1. Let $f_{i} \in W_{0}^{\chi_{j}, 2}(\Omega)$ for all $i, j$ such that $i \neq j$. Then $\|f\|_{V^{1}}^{2}$ is equivalent to the quantity

$$
\begin{aligned}
\inf _{f_{1}+\cdots+f_{n}=f} \sum_{i=1}^{n} \sum_{j: j \neq i}\left\|D_{j} f_{i}\right\|_{\Omega}^{2} & =\pi^{2} \inf _{\tilde{f}_{1, m}+\cdots+\tilde{f}_{n, m}=\tilde{f}_{m} \forall m>0} \sum_{m>0} \sum_{i=1}^{n} \sum_{j: j \neq i} m_{j}^{2} \widetilde{f}_{i, m}^{2} \\
& =\pi^{2} \sum_{m>0}\langle m\rangle_{0}^{2} \widetilde{f}_{m}^{2}
\end{aligned}
$$

with $\langle m\rangle_{0}^{2}=\left(\sum_{i=1}^{n} \sum_{j: j \neq i} m_{j}^{-2}\right)^{-1}$, which is equivalent to $\|f\|_{\mathcal{H}^{1}}^{2}$ if the inequality $(n !)^{-1}\langle m\rangle^{2} \leqslant\langle m\rangle_{0}^{2} \leqslant\langle m\rangle^{2}$ is taken into account.

2 . Let $0 \leqslant \alpha \leqslant 1$. By setting $f^{(M)}=\sum_{\langle m\rangle \leqslant M} \widetilde{f}_{m} \sigma_{m}$, for $M \geqslant 1$, we get

$$
\left\|f-f^{(M)}\right\|_{\mathcal{H}^{0}}^{2}+M^{-2}\left\|f^{(M)}\right\|_{\mathcal{H}^{1}}^{2}=\sum_{m>0} \min \left\{\frac{\langle m\rangle}{M}, 1\right\}^{2} \widetilde{f}_{m}^{2} \leqslant M^{-2 \alpha}\|f\|_{\mathcal{H}^{\alpha}}^{2}
$$

Since by virtue of Lemma 3.1.3 in [4] and the embedding of $\mathcal{H}^{1}$ in $\mathcal{H}^{0}$

$$
\|f\|_{\left(\mathcal{H}^{0}, \mathcal{H}^{1}\right)_{\alpha, \infty}} \leqslant c \sup _{M \geqslant 1}\left[M^{-\alpha} \inf _{\psi \in \mathcal{H}^{1}}\left(\|f-\psi\|_{\mathcal{H}^{0}}^{2}+M^{-2}\|\psi\|_{\mathcal{H}^{1}}^{2}\right)^{1 / 2}\right]
$$

by choosing $\psi=f^{(M)}$ and using the equality $\mathcal{H}^{0}=V^{0}=L^{2}(\Omega)$ along with Claim 1 of the proposition, we obtain inequality (3.3).

Let $\bar{\alpha}=\left(\alpha_{1}, \ldots, \alpha_{n}\right)$ and $\alpha_{i} \geqslant 0$ for all $i$. The following inequality holds:

$$
\|f\|_{\mathcal{H}^{\alpha}} \leqslant 2^{(n-2) \alpha}\|f\|_{S^{\bar{\alpha}} \mathcal{H}} \equiv 2^{(n-2) \alpha}\left(\sum_{m>0} m_{1}^{2 \alpha_{1}} \ldots m_{n}^{2 \alpha_{n}} \widetilde{f}_{m}^{2}\right)^{1 / 2}, \quad \forall \bar{\alpha} \geqslant 0
$$

provided that $\alpha_{1}+\alpha_{2}=\alpha$, for $n=2$, or $\langle\bar{\alpha}\rangle=\alpha$, for $n=3$. This follows from the inequality $\langle m\rangle \leqslant 2^{(n-2) \alpha} m_{1}^{\alpha_{1}} \ldots m_{n}^{\alpha_{n}}$.

The spaces $\mathcal{H}^{\alpha}, S^{\bar{\alpha}} \mathcal{H}$ are Hilbert ones; the spaces $S_{1}^{\chi} \mathcal{H}, S^{\bar{\alpha}} \mathcal{H}$ (for $\bar{\alpha}>0$ ) are spaces of functions having dominating mixed smoothness, cf. [1,2,14]. For $n=2$, the error estimate for $f \in S^{\bar{\alpha}} \mathcal{H}, g=0$, which immediately follows from Theorem 3.1 as well as from inequalities (3.3) and the latter is related to an estimate first discovered (among others) by the author in [21] and developed further in [26] in the hyperbolic case.

By definition of $V^{1}$ it is clear that this space contains some discontinuous functions. Nevertheless, the calculation of the norm $\|f\|_{\mathcal{H}^{1}}$ shows that the space does not contain, for example, the functions $f(x)=\chi_{0}^{i_{1}}\left(x_{1}\right) \ldots \chi_{0}^{i_{n}}\left(x_{n}\right)$, with $\chi_{0}$ being the characteristic function of the interval $\left(\frac{1}{4}, \frac{3}{4}\right)$ and $i_{1}, \ldots, i_{n}$ taking values 0 or 1 . Obviously, for $i_{1}=\cdots=i_{n}=0$, we have simply $f(x) \equiv 1$; otherwise, the function $f$ is discontinuous.

Let $\Pi=\left(a^{(1)}, b^{(1)}\right) \times \cdots \times\left(a^{(n)}, b^{(n)}\right)$ and $\Delta_{(i)}=b^{(i)}-a^{(i)}$. We define the space of functions on $\Pi$ (having dominating mixed derivative $D_{1} \ldots D_{n} f \in L^{1}(\Pi)$ ) equipped with the norm

$$
\|f\|_{S_{1}^{\chi} W(\Pi)}=\sum_{i_{1}=0,1} \ldots \sum_{i_{n}=0,1} \Delta_{(1)}^{i_{1}-1} \ldots \Delta_{(n)}^{i_{n}-1}\left\|D_{1}^{i_{1}} \ldots D_{n}^{i_{n}} f\right\|_{L^{1}(\Pi)} .
$$


Proposition 3.2. 1. The following inequality holds, for $0 \leqslant \alpha<1$ :

$$
\|f\|_{\mathcal{H}^{\alpha}} \leqslant c(1-\alpha)^{-1 / 2}\|f\|_{S_{1}^{\chi} \mathcal{H}}
$$

2. Let $\bar{\Omega}=\cup_{k=1}^{K} \bar{\Pi}^{(k)}$, where $\Pi^{(k)}, 1 \leqslant k \leqslant K$, are the open pairwise nonintersecting parallelepipeds with the edges parallel to the coordinate axes. The following inequality holds:

$$
\|f\|_{S_{1}^{\chi} \mathcal{H}} \leqslant c \sum_{k=1}^{K}\|f\|_{S_{1}^{\chi} W\left(\Pi^{(k)}\right)}
$$

where the constant $c$ depends on $n$ only.

Proof. 1. Clearly, inequality (3.4) holds with the constant $c_{\alpha}\left(\right.$ instead of $\left.c(1-\alpha)^{1 / 2}\right)$ such that

$$
c_{\alpha}^{2}=\sum_{m>0} \frac{\langle m\rangle^{2 \alpha}}{\left(m_{1} \ldots m_{n}\right)^{2}} .
$$

The summands of this series are symmetric functions of $m_{1}, \ldots, m_{n}$, therefore

$$
c_{\alpha}^{2} \leqslant \begin{cases}2 \sum_{m: m_{1} \leqslant m_{2}} m_{1}^{-2 \varepsilon} m_{2}^{-2} & \text { for } n=2, \\ 2^{\alpha} 6 \sum_{m: m_{1} \leqslant m_{2} \leqslant m_{3}} m_{1}^{-2} m_{2}^{-2 \varepsilon} m_{3}^{-2} & \text { for } n=3,\end{cases}
$$

where $\varepsilon=1-\alpha$.

For $n=2$, we bound the series by the integrals which are approximated by this series and find

$$
c_{\alpha}^{2} \leqslant 2 \sum_{m_{1}=1}^{\infty} m_{1}^{-2 \varepsilon} \sum_{m_{2}=m_{1}}^{\infty} m_{2}^{-2} \leqslant 4 \sum_{m_{1}=1}^{\infty} m_{1}^{-1-2 \varepsilon} \leqslant 4\left(1+\frac{1}{2 \varepsilon}\right) .
$$

The case $n=3$ can be reduced to $n=2$ since

$$
c_{\alpha}^{2} \leqslant 12 \sum_{m_{3}=1}^{\infty} \sum_{m_{2}=1}^{m_{3}} m_{2}^{-2 \varepsilon} m_{3}^{-2} \sum_{m_{1}=1}^{m_{2}} m_{1}^{-2} \leqslant 24 \sum_{m_{2}=1}^{\infty} \sum_{m_{3}=m_{2}}^{\infty} m_{2}^{-2 \varepsilon} m_{3}^{-2} \leqslant 48\left(1+\frac{1}{2 \varepsilon}\right) .
$$

2. We write down the formula

$$
m_{1} \ldots m_{n} \widetilde{f}_{m}=2^{n / 2}\left(-\frac{1}{\pi}\right)^{n} \sum_{k=1}^{K} \int_{\Pi^{(k)}} f(x) D_{1} \cos \pi m_{1} x_{1} \cdot \ldots \cdot D_{n} \cos \pi m_{n} x_{n} d x .
$$

Since $\Pi^{(k)}$ can be transformed onto $\Omega$ by an affine change of variables $x_{1}, \ldots, x_{n}$, the formula implies that (3.5) follows from the estimate

$$
|I(w)| \equiv\left|\int_{\Omega} w(x) D_{1} \psi_{1} \cdots D_{n} \psi_{n} d x\right| \leqslant 3^{n}\|w\|_{S_{1}^{\chi} W(\Omega)},
$$

where $\psi_{k}=\psi\left(x_{k}\right)$ and $\psi_{k} \in C^{1}[0,1],\left\|\psi_{k}\right\|_{C[0,1]} \leqslant 1$ for any $k$. 
Estimate (3.6) is proved by induction using integration by parts. Namely, for $n=1$, this is valid. For $n \geqslant 2$, by integrating by parts and applying estimate (3.6) for the dimension $n-1$ we obtain

$$
\begin{aligned}
|I(w)| \leqslant & \left.\left(\left|\int_{\Omega_{\langle n\rangle}} w D_{1} \psi_{1} \cdots D_{n-1} \psi_{n-1} d x_{\langle n\rangle}\right| \psi_{n} \mid\right)\right|_{x_{n}=0} ^{x_{n}=1} \\
& +\int_{0}^{1}\left|\int_{\Omega_{\langle n\rangle}} D_{n} w \cdots D_{1} \psi_{1} D_{n-1} \psi_{n-1} d x_{\langle n\rangle}\right|\left|\psi_{n}\right| d x_{n} \\
\leqslant & 3^{n-1}\left(2\|\| w\left\|_{B_{\langle n\rangle}}\right\|_{C[0,1]}+\|\| D_{n} w\left\|_{B_{\langle n\rangle}}\right\| \|_{L^{1}(0,1)}\right) \\
\leqslant & 3^{n-1}\left(2\|\| w\left\|_{B_{\langle n\rangle}}\right\|_{L^{1}(0,1)}+3\|\| D_{n} w\left\|_{B_{\langle n\rangle}}\right\| \|_{L^{1}(0,1)}\right) \\
\leqslant & 3^{n}\|w\|_{S_{1}^{\chi} W(\Omega)},
\end{aligned}
$$

where $\Omega_{\langle n\rangle}=(0,1)^{n-1}, x_{\langle n\rangle}=\left(x_{1}, \ldots, x_{n-1}\right), B_{\langle n\rangle}=S_{1}^{\chi\langle n\rangle} W\left(\Omega_{\langle n\rangle}\right)$ with $\chi_{\langle n\rangle}=(1, \ldots, 1) \in$ $\mathbb{R}^{n-1}$.

Corollary 3.1. Estimate (3.1) implies the following estimate, for $g=0$ :

$$
\|s u-v\|_{H^{\langle 1\rangle}} \leqslant c|h|^{2}\left|\log h^{(n)}\right|^{1 / 2}\|f\|_{S_{1}^{\chi} \mathcal{H}}
$$

with $h^{(2)}=|h|, h^{(3)}=h_{\min }$. Hereafter in this section, in the estimates with the multipliers $\log h^{(n)}$ it is supposed that $|h| \leqslant \frac{1}{2}$, for $n=2$.

Proof. The estimate is proved by applying inequalities (3.1), (3.3), (3.4) sequentially and choosing $\alpha=\alpha\left(h^{(n)}\right)=1-\left|\log h^{(n)}\right|^{-1}$.

We emphasize that the values of $f$ on different $\Pi^{(k)}$ are not related to each other in inequality (3.5) and thus in Corollary 3.1. Consequently, they include the case of piecewise smooth functions $f$ having discontinuities on the straight lines, for $n=2$, or the planes, for $n=3$, parallel to the coordinate ones. An essential point is that the location of the discontinuities with respect to the mesh $\bar{\omega}^{h}$ is of no importance (in particular, the mesh can be uniform).

3.3. Now we deduce error estimates in terms of the Nikolskii spaces from Theorem 3.1. We define the spaces

$$
V^{\langle\alpha\rangle}=\mathfrak{S} V^{\alpha \chi_{k}}, \quad 0 \leqslant \alpha \leqslant 1,
$$

where

$$
V^{0 \chi_{k}}=L^{2}(\Omega), \quad V^{\alpha \chi_{k}}=H_{0}^{\alpha \chi_{k}, 2}(\Omega), 0<\alpha<1, \quad V^{\chi_{k}}=W_{0}^{\chi_{k}, 2}(\Omega) .
$$

Proposition 3.3. Let $1 \leqslant k \leqslant n$.

1. The space $H_{0}^{\alpha \chi_{k}, 2}(\Omega)$ coincides (up to the equivalence of norms) with the space $H^{\alpha \chi_{k}, 2}(\Omega)$ for $0<\alpha<\frac{1}{2}$ or with the subspace $\left\{w \in H^{\alpha \chi_{k}, 2}(\Omega) ;\left.w\right|_{x_{k}=0}=0\right\}$ for $\frac{1}{2}<\alpha<1$. Moreover, the following inequality holds:

$$
\|w\|_{H_{0}^{\alpha \chi_{k}, 2}(\Omega)} \leqslant c_{\alpha}\|w\|_{H^{\alpha \chi_{k}, 2}(\Omega)}, \quad 0<\alpha<\frac{1}{2}
$$


with $c_{\alpha}=O\left(\alpha^{-1}\left(\frac{1}{2}-\alpha\right)^{-1}\right)$.

2. In the case of $\alpha=\frac{1}{2}$, the following inequalities hold:

$$
\begin{aligned}
\|w\|_{H^{1 / 2 \chi_{k}, 2}(\Omega)} & \leqslant\|w\|_{H_{0}^{1 / 2 \chi_{k}, 2}(\Omega)} \leqslant(1+\sqrt{2})\|w\|_{\left.L^{2}\langle k\rangle\right)_{k}(\Omega)}+\|w\|_{\Omega}^{\left[1 / 2 \chi_{k}\right]} \\
& \leqslant(1+\sqrt{2})\|w\|_{L^{\infty}(\Omega)}+\|w\|_{\Omega}^{\left[1 / 2 \chi_{k}\right]}
\end{aligned}
$$

where the anisotropic norm $\|w\|_{L^{2}\langle k\rangle^{\infty_{k}(\Omega)}}$ differs from $\|w\|_{L^{2}(\Omega)}=\|\ldots\| w\left\|_{L^{2}(0,1)} \ldots\right\|_{L^{2}(0,1)}$ by taking the norm $\|\cdot\|_{L^{\infty}(0,1)}$ instead of $\|\cdot\|_{L^{2}(0,1)}$ with respect to $x_{k}$.

In addition, for $\Omega=\cup_{k=1}^{K} E_{k}$ with $E_{k}, 1 \leqslant k \leqslant K$, being pairwise nonintersecting measurable sets, the following inequality holds:

$$
\|w\|_{\Omega}^{\left[1 / 2 \chi_{k}\right]} \leqslant \sum_{k=1}^{K}\left\{\left[\operatorname{var}_{k} \chi\left(E_{k}\right)\right]^{1 / 2}\|w\|_{L^{\infty}(\Omega)}+\|w\|_{E_{k}}^{[1 / 2]}\right\} .
$$

Here $\operatorname{var}_{k} \chi(E)$ is the variation with respect to $x_{k}$ [18] of the characteristic function $\chi(E)$ of the set $E$.

Proof. 1. The given characterization of the spaces $H_{0}^{\alpha \chi_{k}, 2}(\Omega)$, for $0<\alpha<1, \alpha \neq \frac{1}{2}$, follows from results of $[13,14]$. Moreover, the given estimate for $c_{\alpha}$ can be deduced by following the proofs (though this is cumbersome).

Another way to estimate $c_{\alpha}$ and to verify the coincidence of $H_{0}^{\alpha \chi_{k}, 2}(\Omega)$ and $H^{\alpha \chi_{k}, 2}(\Omega)$, for $0<\alpha<\frac{1}{2}$, is as follows. By the definition of $H_{0}^{\alpha \chi_{k}, 2}(\Omega)$ it is clear that

$$
\begin{aligned}
c_{1}\|w\|_{H_{0}^{\alpha \chi_{k}, 2}(\Omega)} & \leqslant\|w\|_{H^{\alpha \chi_{k}, 2}(\Omega)}+\sup _{0<\gamma<1 / 2} \gamma^{-1 / 2}\left\|\chi_{\gamma}^{(k)} w\right\|_{\Omega} \\
& \leqslant c_{2}\|w\|_{H_{0}^{\alpha \chi_{k}, 2}(\Omega)}, \quad 0<\alpha<1
\end{aligned}
$$

where $\chi_{\gamma}^{(k)}\left(x_{k}\right)=1$ for $x_{k} \in(0, \gamma) \cup(1-\gamma, 1)$; otherwise, $\chi_{\gamma}^{(k)}\left(x_{k}\right)=0$. In addition, obviously we have

$$
\left\|\chi_{\gamma}^{(k)} w\right\|_{\Omega} \leqslant\|w\|_{\Omega}, \quad\left\|\chi_{\gamma}^{(k)} w\right\|_{\Omega} \leqslant(2 \gamma)^{1 / 2}\|w\|_{L^{2\langle k\rangle} \infty_{k}(\Omega)}
$$

therefore, by the interpolation theorem for linear operators

$$
\left\|\chi_{\gamma}^{(k)} w\right\|_{\Omega} \leqslant(2 \gamma)^{\beta / 2}\|w\|_{\left(L^{2}(\Omega), L^{2}\langle k\rangle \infty_{k}(\Omega)\right)_{\beta, \infty}}, \quad 0<\beta<1 .
$$

Since

$$
\|w\|_{L^{2\langle k\rangle} \infty_{k}(\Omega)} \leqslant c\|w\|_{L^{2}(\Omega)}^{1 / 2}\|w\|_{W^{\chi_{k}, 2}(\Omega)}^{1 / 2},
$$

after setting $\alpha=\beta / 2$, we get

$$
\gamma^{-\alpha}\left\|\chi_{\gamma}^{(k)} w\right\|_{\Omega} \leqslant c_{3}\left[\alpha\left(\frac{1}{2}-\alpha\right)\right]^{-1}\|w\|_{\left(L^{2}(\Omega), W \chi_{k}, 2(\Omega)\right)_{\alpha, \infty}}, \quad 0<\alpha<\frac{1}{2} .
$$

Here, we have applied one case of the reiteration theorem, see Section 3.5 in [4], in the following useful form [26].

Let $\left(B_{0}, B_{1}\right)$ be a pair of compatible Banach spaces. If $X_{k}, k=0,1$, are intermidiate spaces with respect to the pair $\left(B_{0}, B_{1}\right)$ such that $\|w\|_{X_{k}} \leqslant \varkappa\|w\|_{B_{0}}^{1-\theta_{k}}\|w\|_{B_{1}}^{\theta_{k}}$ for some $0 \leqslant$ $\theta_{0}<\theta_{1} \leqslant 1$, then

$$
\|w\|_{\left(X_{0}, X_{1}\right)_{\eta(\theta), \infty}} \leqslant c_{0} \frac{\varkappa}{\left(\theta_{1}-\theta\right)\left(\theta-\theta_{0}\right)}\|w\|_{\left(B_{0}, B_{1}\right)_{\theta, \infty}}, \quad \forall \theta \in\left(\theta_{0}, \theta_{1}\right)
$$


with $\eta(\theta)=\left(\theta-\theta_{0}\right) /\left(\theta_{1}-\theta_{0}\right)$.

We complete the proof by taking into account equality (2.6).

Notice that by virtue of (3.10) the functions $w \in H_{0}^{\alpha \chi_{k}, 2}(\Omega)$, for $\alpha>\frac{1}{2}$, clearly have the property $\left.w\right|_{\Gamma_{k}}=0$.

2. Inequalities (3.8) and (3.9) follow from the definition of $\|\cdot\|_{E}^{\left[1 / 2 \chi_{k}\right]}$ (cf. (3.10)); it also should be taken into account that for any bounded measurable set $E \subset \mathbb{R}^{n}$ we have

$$
\gamma^{-1} \text { meas }\left\{E \backslash E_{\gamma \chi_{k}}\right\} \leqslant\left(\|\chi(E)\|_{\mathbb{R}^{n}}^{\left[1 / 2 \chi_{k}\right]}\right)^{2}=\operatorname{var}_{k} \chi(E) ;
$$

the latter equality follows from [10].

Proposition 3.3 along with inequalities (3.10) imply the following. The space $H_{0}^{1 / 2 \chi_{k}, 2}(\Omega)$ is a little bit restricted as against $H^{1 / 2 \chi_{k}, 2}(\Omega)$. Nevertheless, both spaces contain a fairly broad class of discontinuous functions under very weak assumptions on their $(n-1)-$ dimensional surfaces of discontinuities. Namely, if $E$ is a measurable subset in $\Omega$, then the bounded discontinuous function $\chi(E)$ belongs to $H_{0}^{1 / 2 \chi_{k}, 2}(\Omega)$ (or $H^{1 / 2 \chi_{k}, 2}(\Omega)$ ) if and only if $\operatorname{var}_{k} \chi(E)$ is finite taking into account (3.11). On the other hand, if $E$ intersects almost all the straight lines having the direction vector $\chi_{k}$ at no more than $J$ points, then $\operatorname{var}_{k} \chi(E) \leqslant c(J)$, see Section 4.2 in [18]. We emphasize that the values of $w$ on $E_{1}, \ldots, E_{K}$ are not related to each other in inequality (3.9).

Clearly, the space $H_{0}^{1 / 2,2}(\Omega)=\bigcap_{1 \leqslant k \leqslant n} H_{0}^{1 / 2 \chi_{k}, 2}(\Omega)$ contains a broad class of discontinuous functions as well; notice that, for any bounded measurable set $E$, the property $\max _{1 \leqslant k \leqslant n} \operatorname{var}_{k} \chi(E)<\infty$ is equivalent to the finiteness of the perimeter of $E$ [18].

Proposition 3.4. 1. The following inequality holds:

$$
\|w\|_{V^{\alpha}} \leqslant c\|w\|_{V^{\langle\alpha\rangle}}, \quad 0 \leqslant \alpha \leqslant 1 .
$$

2. The space $V^{\langle\alpha\rangle}$ coincides (up to the equivalence of norms) with $\mathfrak{S} H^{\alpha \chi_{k}, 2}(\Omega)$ for $0<$ $\alpha<\frac{1}{2}$ or with $\mathfrak{S} H^{\alpha \chi_{k}, 2}\left(\Omega ; H^{\alpha-1 / 2,2}\left(\Gamma_{k}\right)\right)$ for $\frac{1}{2}<\alpha<1$. Here $H^{\alpha \chi_{k}, 2}\left(\Omega ; H^{\alpha-1 / 2,2}\left(\Gamma_{k}\right)\right)$ is the space of functions $w \in H^{\alpha \chi_{k}, 2}(\Omega)$ having the trace $\left.w\right|_{\Gamma_{k}} \in H^{\alpha-1 / 2,2}\left(\Gamma_{k}\right)$ and equipped with the norm $\max \left\{\|w\|_{H^{\alpha \chi_{k}, 2}(\Omega)},\|w\|_{H^{\alpha-1 / 2,2}\left(\Gamma_{k}\right)}\right\}$.

Proof. Inequality (3.12) follows from equality (2.7).

The given characterization of the spaces $V^{\langle\alpha\rangle}$ is deduced with the aid of Proposition 3.3, Claim 1, as well as with the trace-on-a-coordinate-axis (plane) theorem and its converse for the Nikolskii spaces $H^{\alpha, 2}\left(\mathbb{R}^{n}\right)$ [14].

Corollary 3.2. Estimate (3.1) implies the following error estimates:

$$
\|s u-v\|_{H^{\langle 1\rangle}} \leqslant c \gamma_{n}^{1-\alpha}|h|^{1+\alpha}\left[\|f\|_{V^{\langle\alpha\rangle}}+c_{\alpha}\left(\left\|g_{\Omega}\right\|^{(1)}+\sum_{i=1}^{n}\left\|D_{i}^{2} g_{\Omega}\right\|_{H^{\alpha, 2}(\Omega)}\right)\right]
$$

for $0<\alpha<1, \quad \alpha \neq \frac{1}{2}$

$$
\begin{aligned}
& \|s u-v\|_{H^{\langle 1\rangle}} \leqslant c \gamma_{n}^{1 / 2}|h|^{3 / 2}\left(\|f\|_{V^{\langle 1 / 2\rangle}}+\left\|g_{\Omega}\right\|^{(1)}+\sum_{i=1}^{n}\left\|D_{i}^{2} g_{\Omega}\right\|_{H^{1 / 2,2}(\Omega) \cap V^{\langle 1 / 2\rangle}}\right), \\
& \|s u-v\|_{H^{\langle 1\rangle}} \leqslant c \gamma_{n}^{1 / 2}|h|^{3 / 2}\left|\log h^{(n)}\right|\left(\|f\|_{\mathfrak{S}^{1 / 2 \chi_{k}, 2}(\Omega)}+\left\|g_{\Omega}\right\|^{(1)}+\sum_{i=1}^{n}\left\|D_{i}^{2} g_{\Omega}\right\|_{H^{1 / 2,2}(\Omega)}\right) .
\end{aligned}
$$


The proof of all the estimates follows from Proposition 3.4 (to demonsrate the latter, the estimate for $c_{\alpha}$ from (3.7) should be taken into account). They allow one to follow the growth in order of the convergence rate depending on the smoothness of $f$ and $g$ in detail. The second estimate includes the case of the discontinuous $f$. It is better in order and is given under essentially more broad conditions than the corresponding estimate in [3].

3.4. We set $H_{0}^{\alpha, 2}\left(\Gamma_{k}\right)=H_{0}^{\alpha, 2}\left((0,1)^{n-1}\right) \times H_{0}^{\alpha, 2}\left((0,1)^{n-1}\right)$ taking into account that $\Gamma_{k}$ is a collection of two $(n-1)$-dimensional cubes.

Proposition 3.5. For $0 \leqslant \alpha<2 \beta \leqslant 1$, the following inequality holds:

$$
\|w\|_{\mathcal{H}^{\alpha}} \leqslant c_{\alpha, \beta}\|w\|_{\mathfrak{S} W^{\chi_{k}, 2}\left(\Omega ; H_{0}^{\beta, 2}\left(\Gamma_{k}\right)\right)},
$$

where $c_{\alpha, \beta}=O\left((2 \beta-\alpha)^{-1 / 2}\right)$ and the space $W^{\chi_{k}, 2}\left(\Omega ; H_{0}^{\beta, 2}\left(\Gamma_{k}\right)\right)$ is defined similarly to $H^{\alpha \chi_{k}, 2}\left(\Omega ; H^{\alpha-1 / 2,2}\left(\Gamma_{k}\right)\right)$.

Proof. 1. First, we prove an auxiliary result. We introduce the Fourier sum

$$
z_{(M)}(x)=\sum_{m: m_{1} \leqslant M, \ldots, m_{n} \leqslant M} \widetilde{z}_{m} \sigma_{m}(x),
$$

for $z \in L^{2}(\Omega)$. For any $n \geqslant 1$, the following estimate holds, for $\beta=0,1$ :

$$
\left\|z-z_{(M)}\right\|_{\Omega}+M^{-1}\left\|z_{(M)}\right\|_{H^{\langle 1\rangle}} \leqslant c M^{-\beta}\left\|z_{(M)}\right\|_{H^{\langle\beta\rangle}}
$$

by the interpolation theorem for linear operators the estimate is valid for all $0 \leqslant \beta \leqslant 1$; here, we set $H^{\langle 0\rangle}=L^{2}(\Omega)$ and $H^{\langle\beta\rangle}=\left(L^{2}(\Omega), W_{0}^{1,2}(\Omega)\right)_{\beta, \infty}$ for $0<\beta<1$, for the sake of brevity. In terms of the Fourier coefficients the latter estimate is equivalent to

$$
\left(\sum_{m>0} \gamma_{M}^{2}(m) \widetilde{z}_{m}^{2}\right)^{1 / 2} \leqslant c_{1} M^{-\beta}\|z\|_{H\langle\beta\rangle}
$$

with $\gamma_{M}(m)=\min \left\{\frac{1}{M} \max _{1 \leqslant i \leqslant n} m_{i}, 1\right\}$. By the Hölder inequality we find

$$
\sum_{m>0} \gamma_{M}^{2 \alpha}(m) \widetilde{z}_{m}^{2} \leqslant\left(\sum_{m>0} \gamma_{M}^{2}(m) \widetilde{z}_{m}^{2}\right)^{\alpha}\left(\sum_{m>0} \widetilde{z}_{m}^{2}\right)^{1-\alpha}, \quad 0 \leqslant \alpha \leqslant 1
$$

thus, by taking into account the estimate $\|z\|_{\Omega} \leqslant c\|z\|_{H\langle\beta\rangle}, 0 \leqslant \beta \leqslant 1$, we get

$$
\left(\sum_{m>0} \gamma_{M}^{2 \alpha}(m) \widetilde{z}_{m}^{2}\right)^{1 / 2} \leqslant c_{2} M^{-\beta \alpha}\|z\|_{H^{\langle\beta\rangle}}, \quad \alpha, \beta \in[0,1] .
$$

2. Let $\alpha, \beta \in(0,1)$. We set $B^{(k)}=W^{\chi_{k}, 2}\left(\Omega ; H_{0}^{\beta, 2}\left(\Gamma_{k}\right)\right)$. Clearly, inequality (3.13) follows from the following one, for $1 \leqslant i \leqslant n$ :

$$
\|w\|_{\mathcal{H}^{\alpha}} \leqslant c_{\alpha, \beta}\|w\|_{\cap_{1 \leqslant k \leqslant n, k \neq i} B^{(k)}} .
$$

By exploiting the symmetry of variables it suffices to prove the inequality, for example, for $i=1$. 
Integration by parts gives the formula, for $m_{\langle n\rangle}=\left(m_{1}, \ldots, m_{n-1}\right)$,

$$
-\pi m_{n} \widetilde{w}_{m}=\sqrt{2}(-1)^{m_{n}} \widetilde{w}_{m_{\langle n\rangle}}^{(1)}-\sqrt{2} \widetilde{w}_{m_{\langle n\rangle}}^{(0)}-{\widetilde{D_{n} w_{m,(n)}}}
$$

where on the right-hand side we use the Fourier coefficients of the functions $w^{(k)}=$ $\left.w\right|_{x_{n}=k}, k=0,1$, and $D_{n} w$ with respect to the systems of functions differing from $\sigma_{m}(x)$ by omitting the cofactor $\sqrt{2} \sin \pi m_{n} x_{n}$ and replacing it by $\sqrt{2} \cos \pi m_{n} x_{n}$, respectively.

For $n=2$, by virtue of (3.16) and the inequality $\langle m\rangle=m_{2} \gamma_{m_{2}}\left(m_{1}\right) \leqslant m_{2}$ we can write down

$$
\begin{aligned}
\|w\|_{\mathcal{H}^{\alpha}} & \leqslant \sum_{k=0,1}\left[\sum_{m>0}\langle m\rangle^{2 \alpha} m_{2}^{-2}\left(\widetilde{w}_{m_{1}}^{(k)}\right)^{2}\right]^{1 / 2}+\left(\sum_{m>0}\langle m\rangle^{2 \alpha} m_{2}^{-2}{\widetilde{D_{2}}}_{m,(2)}^{2}\right)^{1 / 2} \\
& \leqslant \sum_{k=0,1}\left[\sum_{m_{2}=1}^{\infty} m_{2}^{2(\alpha-1)} \sum_{m_{2}=1}^{\infty} \gamma_{m_{2}}^{2 \alpha}\left(m_{1}\right)\left(\widetilde{w}_{m_{1}}^{(k)}\right)^{2}\right]^{1 / 2}+\left(\sum_{m>0}{\widetilde{D_{2}}}_{m,(2)}^{2}\right)^{1 / 2} .
\end{aligned}
$$

Let $2 \alpha(1-\beta)<1$. By applying inequality (3.14) and taking into account equality (2.9) (both in the 1D-case), we get

$$
\|w\|_{\mathcal{H}^{\alpha}} \leqslant c\left[1+(2 \beta \alpha-2 \alpha+1)^{-1}\right]^{1 / 2} \sum_{k=0,1}\left\|w^{(k)}\right\|_{H_{0}^{\beta, 2}(0,1)}+\left\|D_{2} w\right\|_{\Omega} .
$$

For $n=3$, we first write down the decomposition

$$
w=w_{1}+w_{2}=\sum_{m: m_{2} \leqslant m_{3}} \widetilde{w}_{m} \sigma_{m}+\sum_{m: m_{2}>m_{3}} \widetilde{w}_{m} \sigma_{m}
$$

By virtue of (3.16) and by analogy with (3.17) we can write down (since $\langle m\rangle \leqslant$ $2 m_{3} \gamma_{m_{3}}\left(m_{\langle 3\rangle}\right) \leqslant 2 m_{3}$ for $\left.m_{2} \leqslant m_{3}\right)$

$$
\begin{aligned}
\left\|w_{1}\right\|_{\mathcal{H}^{\alpha}} \leqslant & \sum_{k=0,1}\left[\sum_{m_{3}=1}^{\infty} m_{3}^{2(\alpha-1)} \sum_{m_{2}=1}^{m_{3}} \sum_{m_{1}=1}^{\infty} \gamma_{m_{3}}^{2 \alpha}\left(m_{\langle 3\rangle}\right)\left(\widetilde{w}_{m_{\langle 3\rangle}}^{(k)}\right)^{2}\right]^{1 / 2} \\
& +\left(\sum_{m_{3}=1}^{\infty} \sum_{m_{2}=1}^{m_{3}} \sum_{m_{1}=1}^{\infty} \widetilde{D_{3} w_{m,(3)}^{2}}\right)^{1 / 2} .
\end{aligned}
$$

By enlarging the sum limits with respect to $m_{2}$ from 1 to $\infty$ and applying inequality (3.14) in the $2 \mathrm{D}$-case, we obtain

$$
\left\|w_{1}\right\|_{\mathcal{H}^{\alpha}} \leqslant c\left[1+(2 \beta \alpha-2 \alpha+1)^{-1}\right]^{1 / 2} \sum_{k=0,1}\left\|w^{(k)}\right\|_{H_{0}^{\beta, 2}\left(\Omega_{\langle 3\rangle}\right)}+\left\|D_{3} w\right\|_{\Omega} .
$$

The similar estimate for $w_{2}$ is deduced with the aid of the formula of the type of (3.16) with $D_{2} w$ instead of $D_{3} w$. Since $2 \beta-\alpha \leqslant 2 \beta \alpha-2 \alpha+1$ for $2 \beta \leqslant 1$, inequality (3.15) is proved.

Corollary 3.3. The following error estimate holds:

$$
\|s u-v\|_{H^{\langle 1\rangle}} \leqslant c|h|^{2}\left|\log h^{(n)}\right|^{\sigma}\left(\|f\|_{\mathfrak{S} B^{(k)}}+\left\|g_{\Omega}\right\|^{(1)}+\sum_{i=1}^{n} \sum_{\substack{1 \leqslant j \leqslant n, j \neq i}}\left\|D_{i}^{2} g_{\Omega}\right\|_{B^{(j)}}\right),
$$

where either $\sigma=\frac{1}{2}, B^{(k)}=W^{\chi_{k}, 2}\left(\Omega ; H_{0}^{1 / 2,2}\left(\Gamma_{k}\right)\right)$ or $\sigma=\frac{3}{2}, B^{(k)}=W^{\chi_{k}, 2}\left(\Omega ; H^{1 / 2,2}\left(\Gamma_{k}\right)\right)$. 
Proof. By virtue of Theorem 3.1 and inequality (3.3) along with Theorem 2.1 we get, for $0 \leqslant \alpha \leqslant 1$,

$$
\|s u-v\|_{H^{\langle 1\rangle}} \leqslant c \gamma_{n}^{1-\alpha}|h|^{1+\alpha}\left\|f-L g_{\Omega}\right\|_{\mathcal{H}^{\alpha}}+c|h|^{2} \sum_{i=1}^{n} \sum_{j: j \neq i}\left\|D_{i}^{2} g_{\Omega}\right\|_{W^{\chi_{j}, 2}(\Omega)} .
$$

Set $\alpha=1-\left|\log h^{(n)}\right|^{-1}$. We apply inequality (3.13), for $\beta=\frac{1}{2}$, in the case of $\sigma=\frac{1}{2}$ or sequentially inequalities (3.13), for $\beta=\frac{1}{2}\left(1-\frac{1}{2}\left|\log h^{(n)}\right|^{-1}\right)$, and (3.7), with $\beta$ substituted for $\alpha$, in the case of $\sigma=\frac{3}{2}$. By taking into account the inequality

$$
\left\|L g_{\Omega}\right\|_{\mathfrak{S} B^{(k)}} \leqslant c\left(\left\|g_{\Omega}\right\|^{(1)}+\sum_{i=1}^{n} \sum_{j: j \neq i}\left\|D_{i}^{2} g_{\Omega}\right\|_{B^{(j)}}\right)
$$

(for the proof see below) as well, we obtain estimate (3.18).

To prove estimate (3.19), we first apply the inequality

$$
\left\|L g_{\Omega}\right\|_{\mathfrak{S} B^{(k)}} \leqslant \sum_{i=1}^{n} \sum_{j: j \neq i}\left(\left\|a_{i i} D_{i}^{2} g_{\Omega}\right\|_{B^{(j)}}+\left\|\left(b_{i}-D_{i} a_{i i}\right) D_{i} g_{\Omega}\right\|_{B^{(j)}}\right)+\sum_{j=1}^{n}\left\|a g_{\Omega}\right\|_{B^{(j)}} .
$$

In addition clearly

$$
\left\|g_{\Omega}\right\|_{W^{2,2}(\Omega)}+\sum_{j=1}^{n}\|g\|_{W^{2,2}\left(\Gamma_{j}\right)}+\|g\|_{C(\partial \Omega)} \leqslant c\left(\left\|g_{\Omega}\right\|^{(1)}+\sum_{i=1}^{n} \sum_{j: j \neq i}\left\|D_{i}^{2} g_{\Omega}\right\|_{W^{\chi_{j}, 2}(\Omega)}\right) .
$$

By using the conditions on the coefficients of $L$, the inequalities

$$
\|v w\|_{H^{1 / 2,2}\left(\Gamma_{j}\right)} \leqslant c\|v\|_{C^{1 / 2}\left(\bar{\Gamma}_{j}\right)}\|w\|_{H^{1 / 2,2}\left(\Gamma_{j}\right)}
$$

(where $C^{1 / 2}\left(\bar{\Gamma}_{j}\right)$ is a Hölder space, for example see [11]) and (3.10) along with the embeddings of $W^{1,2}(\Omega)$ in $W^{1 / 2,2}(\partial \Omega)$ and $W^{1 / 2,2}\left(\Gamma_{j}\right)$ in $H^{1 / 2,2}\left(\Gamma_{j}\right)$, we obtain inequality (3.19).

Remark 3.1. The sum of the norms of $f$ and $g$ on the right-hand side of estimate (3.18) can be certainly bounded by

$$
c\left(\|f\|_{W^{1,2}\left(\Omega ; L^{\infty}(\partial \Omega)\right)}+\left\|g_{\Omega}\right\|_{W^{3,2}(\Omega)}+\|g\|_{W^{2, \infty}(\partial \Omega)}\right), c\left(\|f\|_{W^{1,2}(\Omega)}+\left\|g_{\Omega}\right\|_{W^{3,2}(\Omega)}\right)
$$

in the cases of $B^{(k)}=W^{\chi_{k}, 2}\left(\Omega ; H_{0}^{1 / 2,2}\left(\Gamma_{k}\right)\right), W^{\chi_{k}, 2}\left(\Omega ; H^{1 / 2,2}\left(\Gamma_{k}\right)\right)$, respectively. Here, $W^{1,2}\left(\Omega ; L^{\infty}(\partial \Omega)\right)$ is the space of functions $w \in W^{1,2}(\Omega)$ having the trace $\left.w\right|_{\partial \Omega} \in L^{\infty}(\partial \Omega)$ and equipped with the norm $\max \left\{\|w\|_{W^{1,2}(\Omega)},\|w\|_{L^{\infty}(\partial \Omega)}\right\}$.

In Corollary 3.3 we have removed the compatibility condition between $f$ and $g$ at the vertices of $\Omega$ for $n=2$ or on the edges of $\Omega$ for $n=3$, which was implicitly imposed in estimate (3.1) for $\alpha=1$. Theorem 2.2 implies that the compatibility condition must hold in all the error estimates proved under the condition that $u \in W^{3,2}(\Omega)$, in particular, see $[7,8,12,16]$. In practice, such compatibility conditions are very burdensome. 


\section{Lower error estimates}

In this section, we show that all the error estimates without the logarithmic multipliers proved in Section 3 are sharp in order for the method under consideration and moreover they cannot be strengthened for substantially more restricted spaces of the right-hand sides.

Let $C^{\lambda}(\bar{\Omega}), 0<\lambda<1$, and $C^{\ell}(\bar{\Omega}), \ell \geqslant 0$, be the Hölder spaces of continuous, on $\bar{\Omega}$, functions satisfying the Hölder condition of order $\lambda$ on $\bar{\Omega}$ and having continuous, on $\bar{\Omega}$, derivatives of order up to $\ell$, for example see [11]. Let also $C^{\ell \chi_{k}}(\bar{\Omega})$ be the anisotropic space of functions equipped with the norm $\sum_{j=0}^{\ell}\left\|D_{k}^{j} w\right\|_{C^{0}(\bar{\Omega})}$. Next, let $C_{0}^{\lambda}(\bar{\Omega}), C_{0}^{\ell}(\bar{\Omega}), C_{0}^{\ell \chi_{k}}(\bar{\Omega})$ be the subspaces of the just introduced spaces consisting of the functions $w$ which satisfy the boundary conditions, respectively

$$
\left.w\right|_{\partial \Omega}=0 ;\left.\quad D_{\mathbf{n}}^{2 j} w\right|_{\partial \Omega}=0,0 \leqslant 2 j \leqslant \ell ;\left.\quad D_{k}^{2 j} w\right|_{\partial \Omega}=0,0 \leqslant 2 j \leqslant \ell
$$

with $\mathbf{n}$ being the normal to $\partial \Omega$.

Definition 4.1. Let $g=0$. The error of the method (in the mesh counterpart of norm in $W^{1,2}(\Omega)$ ) on a Banach space $B \subset L^{2}(\Omega)$ of the right-hand sides $f$ is the quantity

$$
\mathcal{E}(B)=\sup _{f \in B} \frac{\|s u-v\|_{H^{\langle 1\rangle}}}{\|f\|_{B}}
$$

Theorem 4.1. Let $n=2,3$ and $L=-\varkappa_{i} D_{i}^{2}$ with $\varkappa_{i}$ being constants such that $N^{-1} \leqslant$ $\varkappa_{i} \leqslant N$. Let the mesh $\bar{\omega}^{h}$ be uniform and $|h| \leqslant h_{0}$, for a sufficiently small $h_{0}>0$. Then the following lower estimates are valid:

1. for $B_{\alpha}=C_{0}^{\alpha}(\bar{\Omega}), \bigcap_{k=1}^{n} V^{\alpha \chi_{k}}, \bigcap_{k=1}^{n} S^{\alpha \chi_{k}} \mathcal{H}$ and all the more for $B_{\alpha}=V^{\alpha}, V^{\langle\alpha\rangle}, \mathcal{H}^{\alpha}$,

$$
\mathcal{E}\left(B_{\alpha}\right) \geqslant c_{1}|h|^{1+\alpha}, 0 \leqslant \alpha \leqslant 1
$$

2. $\|s u-v\|_{H^{\langle 1\rangle}} \geqslant c_{0}|h|^{2}$, for $f(x)=\sin \pi x_{1} \ldots \sin \pi x_{n}$, and all the more $\mathcal{E}\left(C_{0}^{\ell}(\bar{\Omega})\right) \geqslant c_{\ell}|h|^{2}$ for all $\ell \geqslant 1$;

3. in the case of $n=3,1 \leqslant k \leqslant n$, and $\ell \geqslant 1$,

$$
\mathcal{E}\left(C_{0}^{\ell \chi_{k}}(\bar{\Omega})\right) \geqslant c_{\ell}|h|
$$

provided that $h_{k} \geqslant N^{-1}|h|$.

Proof. For $f(x)=\sigma_{m}(x)=2^{n / 2} \sin \pi m_{1} x_{1} \ldots \sin \pi m_{n} x_{n}$ with $1 \leqslant m_{k} \leqslant N_{k}-1$ for all $k$, the following explicit formulas hold:

$$
u=\frac{1}{\pi^{2}|m|_{\varkappa}^{2}} \sigma_{m}, \quad v=\frac{a^{(1)} \ldots a^{(n)}}{\lambda_{m}} s \sigma_{m}
$$

under the hypotheses of the theorem. Here

$$
|m|_{\varkappa}^{2}=\sum_{i=1}^{n} \varkappa_{i} m_{i}^{2}, \quad \lambda_{m}=\pi^{2} \sum_{i=1}^{n} \varkappa_{i} m_{i}^{2} \beta^{\langle i\rangle} a^{(i)}
$$


with

$$
\beta^{\langle i\rangle}=\prod_{k: k \neq i} \beta^{(k)}, \quad \beta^{(k)}=1-\frac{2}{3} \sin ^{2} \xi^{(k)}, \quad a^{(k)}=\left(\frac{\sin \xi^{(k)}}{\xi^{(k)}}\right)^{2}, \quad \xi^{(k)}=\frac{\pi m_{k} h_{k}}{2}
$$

Notice that $a^{(k)}>\beta^{(k)}$ (by virtue of the inequality $\left(\frac{\sin \xi}{\xi}\right)^{2}>1-\frac{2}{3} \sin ^{2} \xi$, for $0<\xi \leqslant \frac{\pi}{2}$ ). By taking into account the formula $\left\|s \sigma_{m}\right\|_{H^{\langle 1\rangle}}^{2}=\lambda_{m}$, we find

$$
\|s u-v\|_{H^{\langle 1\rangle}}^{2}=\sum_{i=1}^{n} \frac{\varkappa_{i} m_{i}^{2}}{\lambda_{m}^{1 / 2}|m|_{\varkappa}^{2}}\left(a^{\langle i\rangle}-\beta^{\langle i\rangle}\right) a^{(i)} \text { with } a^{\langle i\rangle}=\prod_{k: k \neq i} a^{(k)} .
$$

Clearly, for example,

$$
a^{\langle 1\rangle}-\beta^{\langle 1\rangle}= \begin{cases}a^{(2)}-\beta^{(2)} & \text { for } n=2, \\ \left(a^{(2)}-\beta^{(2)}\right) a^{(3)}+\beta^{(2)}\left(a^{(3)}-\beta^{(3)}\right) & \text { for } n=3 .\end{cases}
$$

We apply the inequalities $\left(\frac{2}{\pi}\right)^{2}<a^{(k)}<1, \frac{1}{3}<\beta^{(k)}<1$ along with the expansions

$$
a^{(k)}-\beta^{(k)}=\left(\frac{2}{\pi}\right)^{2}-\frac{1}{3}+O\left(h_{k}\right) \text { or } a^{(k)}-\beta^{(k)}=\frac{h_{k}^{2}}{3}\left(1+O\left(h_{k}\right)\right),
$$

for $m_{k}=N_{k}-1$ or $m_{k}=1$, respectively. To prove Claims 1, 2, and 3, it suffices to choose $m_{1}=\cdots=m_{n}=\min _{k} N_{k}-1, \quad m_{1}=\cdots=m_{n}=1$, and $m_{k}=1, m_{l}=\min _{j: j \neq k} N_{j}-1$, for $l \neq$ $k$, respectively.

Remark 4.1. The theorem remains valid if one restricts $\|s u-v\|_{H^{\langle 1\rangle}}$ up to $\| s u-$ $v \|_{W^{1,1}(\Omega)}$.

According to Claim 1, estimate (3.1) along with the first and second ones in Corollary 3.2 are sharp in order (provided that $|h| \leqslant N h_{\min }$ for $n=3$ ). Moreover, these estimates cannot be strengthened even if the smoothness of $f$ of order $\alpha$ is considered in the essentially more restrictive classical sense (i.e., the Hölder one) rather than in the Sobolev or Nikolskii sense. By virtue of Claim 2 the error estimate of the order higher than $c|h|^{2}$ cannot be proved under arbitrarily high smoothness of $f$. According to Claim 3, in the case of $n=3$, an arbitrarily high smoothness of $f$ with respect to only one of variables $x_{1}, \ldots, x_{n}$ cannot guarantee the error estimate of the order higher than $c|h|$ (in contrast to the case $n=2$ ).

\section{Superconvergence of a gradient in the case of $2 \mathrm{D}$ linear finite elements}

Error estimates of the type established in Sections 2 and 3 can be derived for other methods as well (in general with some restrictions and by another technique). Let us consider the case of very well-known linear elements, for an example see $[6,15]$.

We confine ourselves to the $2 \mathrm{D}$ case (i.e., $n=2$ ). Let the mesh $\bar{\omega}^{h}$ be uniform. We divide all the rectangles $\Pi \in \mathcal{P}_{h}$ by two triangles by leading the diagonals parallel to the vector $\left(h_{1}, h_{2}\right)$. We introduce the space $\widehat{S}$ of functions continuous on $\bar{\Omega}$ and linear on any triangle of the partition of $\bar{\Omega}$. We denote by $\widehat{u}$ the piecewise linear filling of the function $u \in C(\bar{\Omega})$, i.e., the element in $\widehat{S}$ coinciding with $u$ at all the nodes of $\bar{\omega}^{h}$. 
Let the boundary-value problem (2.1), (2.2) satisfy the conditions from Section 2.1 and $f \in L^{2}(\Omega), g_{\Omega} \in W^{2,2}(\Omega)$. In this section, we consider as an approximate solution the function $v \in \widehat{S}$ satisfying the integral identity

$$
\mathcal{L}_{\Omega}(v, \varphi)=\int_{\Omega} f \varphi d x, \quad \forall \varphi \in \widehat{S},\left.\varphi\right|_{\partial \Omega}=0
$$

and the mesh boundary condition $v=g$ on $\partial \omega^{h}$. Concerning the validity of inequality of the type of (2.10) with $S^{(1)}$ replaced by $\left\{\varphi \in \widehat{S} ;\left.\varphi\right|_{\partial \Omega}=0\right\}$, see Section 4.2 in [15] (in general, the condition $|h| \leqslant h_{0}$ is required).

Let $D w=\left(D_{1} w, D_{2} w\right), D^{2} w=\left\{D_{i} D_{j} w\right\}_{i, j=1}^{2}$ and, for $k=1,2$, a norm of $D^{k} w$ be the sum of the norms of the elements of $D^{k} w$.

Theorem 5.1. Let $\|\operatorname{div} \mathbf{b}\|_{L^{\infty}(\Omega)} \leqslant N$ and $0 \leqslant \alpha \leqslant 1$. The following a priori error estimate holds:

$$
\|\widehat{u}-v\|_{H^{\langle 1\rangle}} \leqslant c|h|^{1+\alpha}\left\|D^{2} u\right\|^{(\alpha)} .
$$

In the case of $a_{i j}=0$ for all $i \neq j$, the estimate

$$
\|\widehat{u}-v\|_{H^{\langle 1\rangle}} \leqslant c|h|^{2}\left(\sum_{i=1,2}\left\|D_{i}^{2} u\right\|_{\Omega}+\left\|D_{1} D_{2} u\right\|^{(1)}\right)
$$

and more generally the estimate

$$
\|\widehat{u}-v\|_{H^{\langle 1\rangle}} \leqslant c|h|^{1+\alpha}\left(\sum_{i=1,2} \sum_{j: j \neq i}\left\|D_{i}^{2} u\right\|^{\left(\alpha \chi_{j}\right)}+\left\|D_{1} D_{2} u\right\|^{(\alpha)}\right)
$$

are valid.

Proof. 1. For $w \in W^{2,2}(\Omega)$, the following estimates for the error of approximation hold, see Section 2.3.2 in [15]:

$$
\|w-\widehat{w}\|_{\Omega} \leqslant c|h|^{2}\left\|D^{2} w\right\|_{\Omega}, \quad\left\|D_{i}(w-\widehat{w})\right\|_{\Omega} \leqslant c|h|\left\|D D_{i} w\right\|_{\Omega}, \quad i=1,2 .
$$

Let $\varphi \in \widehat{S},\left.\varphi\right|_{\partial \Omega}=0$. The error $r=\widehat{u}-v$ satisfies the identity

$$
\mathcal{L}_{\Omega}(r, \varphi)=-\sum_{i=1,2} \mathcal{L}_{i, \Omega}(u-\widehat{u}, \varphi)-\mathcal{L}_{0, \Omega}(u-\widehat{u}, \varphi)
$$

where, for $k=1,2$,

$$
\mathcal{L}_{k, \Omega}(w, \varphi)=\sum_{j=1,2} \int_{\Omega} a_{k j} D_{j} w D_{k} \varphi d x, \mathcal{L}_{0, \Omega}(w, \varphi)=\int_{\Omega}\left(\sum_{i=1,2} b_{i} D_{i} w+a w\right) \varphi d x .
$$

The proof of the error estimates is reduced to deriving estimates for the bilinear forms. The estimate

$$
\mathcal{L}_{0, \Omega}(u-\widehat{u}, \varphi) \leqslant c|h|^{2}\left\|D^{2} u\right\|_{\Omega}\|\varphi\|_{H^{\langle 1\rangle}}
$$

is proved in the same manner as in the proof of Theorem 2.1. Now, we prove the estimate, for $0 \leqslant \alpha \leqslant 1$,

$$
\mathcal{L}_{k, \Omega}(u-\widehat{u}, \varphi) \leqslant c|h|^{1+\alpha}\left\|D^{2} u\right\|^{(\alpha)}\|\varphi\|_{H^{\langle 1\rangle}}, \quad k=1,2 .
$$


For $\alpha=0$, the estimate immediately follows from (5.4). We recall the proof for $\alpha=1$, see Section 4.3 in [15]. Let $k=1$ for definiteness. Let $p_{l-\frac{1}{2}, m}$ be the open parallelogram consisting of two triangles from the partition of $\bar{\Omega}$ having the common side $\left\{\left(x_{1}, x_{2}\right) ; x_{1} \in\right.$ $\left.\Delta_{1, l-\frac{1}{2}}, x_{2}=x_{2, m}\right\}$. We denote by $\Omega^{(1)}$ the union of all such parallelograms in $\Omega$. For $w \in L^{1}\left(\Omega^{(1)}\right)$, we introduce the piecewise constant function

$$
\langle w\rangle(x)=\langle w\rangle_{1}(x) \equiv\left(h_{1} h_{2}\right)^{-1} \int_{p_{l-\frac{1}{2}}, m} w(\xi) d \xi \quad \text { for } x \in p_{l-\frac{1}{2}, m},
$$

for all $l, m$. Let the functions $\bar{a}_{1 j}$ be constant and, for example, equal $\left\langle a_{1 j}\right\rangle$ on each $p_{l-\frac{1}{2}}, m$. Since $D_{1} \varphi$ is constant on each $p_{l-\frac{1}{2}, m}$ and equals 0 on $\Omega \backslash \bar{\Omega}^{(1)}$, we get

$$
\begin{aligned}
\mathcal{L}_{1, \Omega}(u-\widehat{u}, \varphi)= & \sum_{j=1,2}\left(\int_{\Omega^{(1)}} \bar{a}_{1 j}\left\langle D_{j}(u-\widehat{u})\right\rangle D_{1} \varphi d x\right. \\
& \left.+\int_{\Omega^{(1)}}\left(a_{1 j}-\bar{a}_{1 j}\right) D_{j}(u-\widehat{u}) D_{1} \varphi d x\right) .
\end{aligned}
$$

The second summand is bounded by $c|h|^{2}\left\|D a_{1 j}\right\|_{L^{\infty}(\Omega)}\left\|D D_{j} u\right\|_{\Omega}\|\varphi\|_{H^{(1)}}$. In addition, it follows from [15], pp. 186, 187 that the following estimate holds:

$$
\left\|\left\langle D_{j}(u-\widehat{u})\right\rangle\right\|_{\Omega} \leqslant c|h|^{2}\left\|D^{2} u\right\|^{(1)}, \quad j=1,2 .
$$

Therefore (5.5) is valid for $\alpha=1, k=1$.

Now, we fix the function $\varphi$ in (5.5) and consider the left-hand side as a linear functional on the space $W^{2,2}(\Omega)$. By applying the same interpolation argument as in the proof of estimate (2.13) in Theorem 2.1, we establish (5.5) for any $0 \leqslant \alpha \leqslant 1$. Estimate (5.1) is proved.

2. The above argument shows that in order to prove (5.2) in the case $a_{12}=a_{21}=0$ it suffices to strengthen (5.6), for $j=1$, up to the estimate

$$
\left\|\left\langle D_{1}(u-\widehat{u})\right\rangle\right\|_{\Omega} \leqslant c|h|^{2}\left(\left\|D_{1} D_{2}^{2} u\right\|_{\Omega}+\left\|D_{1}^{2} D_{2} u\right\|_{\Omega}\right),
$$

since it implies the estimate

$$
\mathcal{L}_{k, \Omega}(u-\widehat{u}, \varphi) \leqslant c|h|^{2}\left(\left\|D_{k}^{2} u\right\|_{\Omega}+\left\|D_{1} D_{2} u\right\|^{(1)}\right)\|\varphi\|_{H^{\langle 1\rangle}}, \quad k=1,2,
$$

instead of (5.5).

Let us establish estimate (5.7). We denote by $p$ the reference parallelogram having the vertices $\left( \pm \frac{1}{2}, 0\right),\left(\frac{1}{2}, 1\right),\left(-\frac{1}{2},-1\right)$ and by $p^{*}$ the symmetric one with respect to the ordinate axis. We consider the linear functionals

$$
\ell_{ \pm}(w)=\frac{1}{2}\left(\int_{p} w d x \pm \int_{p^{*}} w d x\right)
$$

The following formula holds:

$$
\ell_{+}(w)=\frac{1}{2} \int_{-1}^{1} \int_{\Delta_{0}} \varkappa w d x_{1} d x_{2} \text { with } \quad \frac{1}{2} \int_{-1}^{1} \varkappa\left(x_{1}, x_{2}\right) d x_{2}=1,
$$


where $\Delta_{0}=\left(-\frac{1}{2}, \frac{1}{2}\right)$ and $\varkappa$ is a piecewise constant function (taking values $\left.0,1,2\right)$, even with respect to $x_{2}$ (and $\left.x_{1}\right)$. Further

$$
\begin{aligned}
\ell_{-}(w) & =\int_{t}\left[w\left(x_{1}, x_{2}\right)-w\left(-x_{1}, x_{2}\right)+w\left(-x_{1},-x_{2}\right)-w\left(x_{1},-x_{2}\right)\right] d x \\
& =\int_{t} \int_{\pi(x)} D_{1} D_{2} w(\xi) d \xi d x \quad \text { with } \pi(x)=\left[-x_{1}, x_{1}\right] \times\left[-x_{2}, x_{2}\right],
\end{aligned}
$$

where $t$ is the triangle with the vertices $\left(0, \frac{1}{2}\right),\left(\frac{1}{2}, 0\right),\left(\frac{1}{2}, 1\right)$. Now, it is clear that

$$
\begin{aligned}
\left|\int_{p} w d x-\int_{\Delta_{0}} w\left(x_{1}, 0\right) d x_{1}\right| & \leqslant\left|\ell_{+}(w)-\int_{\Delta_{0}} w\left(x_{1}, 0\right) d x_{1}\right|+\left|\ell_{-}(w)\right| \\
& \leqslant c_{0} \int_{\Delta_{0} \times(-1,1)}\left(\left|D_{2}^{2} w\right|+\left|D_{1} D_{2} w\right|\right) d x .
\end{aligned}
$$

Since $\left\langle D_{1}(u-\widehat{u})\right\rangle(x)=\left\langle D_{1} u\right\rangle(x)-\left[D_{1} u\left(\cdot, x_{2, m}\right)\right]_{1}^{h}$ for $x \in p_{l-\frac{1}{2}}, m$, the standard argument (of taking the affine transformation of $p_{l-\frac{1}{2}, m}$ onto $p$ ) allows one to derive estimate (5.7) from (5.9).

3. It remains to prove estimate (5.3). This is a consequence of the following one, for $0 \leqslant \alpha \leqslant 1$,

$$
\mathcal{L}_{k, \Omega}(u-\widehat{u}, \varphi) \leqslant c|h|^{1+\alpha}\left(\left\|D_{k}^{2} u\right\|^{\left(\alpha \chi_{\ell}\right)}+\left\|D_{1} D_{2} u\right\|^{\left(\alpha \chi_{\ell}\right)}\right)\|\varphi\|_{H^{\langle 1\rangle}}, \quad k=1,2,
$$

where $\ell \neq k$. For $\alpha=0$, the estimate follows from the definition of $\mathcal{L}_{k, \Omega}$ and (5.4), whereas for $\alpha=1$, it is the same as (5.8). We again fix $\varphi$ in (5.10) and consider its left-hand side as a linear functional on $W^{2,2}(\Omega)$. Once again by applying the interpolation argument from the proof of Theorem 2.1, but using the extension and mollification of $u$ with respect to $x_{\ell}$ only and also applying the equality $\left(L^{2}(\Omega), W^{\chi_{\ell}, 2}(\Omega)\right)_{\alpha, \infty}=H^{\chi_{\ell}, 2}(\Omega)$, see (2.6), we prove (5.10) for $0<\alpha<1$.

Let us consider the case of the pointwise approximation of the leading coefficients of $L$ (instead of the above integral one). For $w \in C(\bar{\Omega})$, we denote by $(w)_{1}$ the function which is constant on each $p_{l-\frac{1}{2}}, m$ and equal either to the value of $w$ at the middle of the, parallel to $\chi_{1}$, diagonal of $p_{l-\frac{1}{2}}, m$ or to the half-sum of the values of $w$ at its ends. Let $q_{l, m-\frac{1}{2}}$ be the open parallelogram consisting of two triangles from the partition of $\bar{\Omega}$ having the common side $\left\{\left(x_{1}, x_{2}\right) ; x_{1}=x_{1, l}, x_{2} \in \Delta_{2, m-\frac{1}{2}}\right\}$. The quantities $\Omega^{(2)},\langle w\rangle_{2},(w)_{2}$ are defined in the same way as $\Omega^{(1)},\langle w\rangle_{1},(w)_{1}$, but by being based on $q_{l, m-\frac{1}{2}}$ instead of $p_{l-\frac{1}{2}, m}$. We introduce the bilinear form

$$
(\mathcal{L})_{\Omega}(v, \varphi)=\sum_{i, j=1,2} \int_{\Omega^{(i)}}\left(a_{i j}\right)_{i} D_{j} v D_{i} \varphi d x+\mathcal{L}_{0, \Omega}(v, \varphi)
$$

and exploit this form instead of $\mathcal{L}_{\Omega}(v, \varphi)$ to define the approximate solution. 
Corollary 5.1. Let $\left\|D^{2} a_{i j}\right\|_{L^{\infty}(\Omega)} \leqslant N$ for all $i, j$ and $|h| \leqslant h_{0}$. Then, for the above modified method with the pointwise approximation of $a_{i j}$, Theorem 5.1 remains valid provided that in (5.1)-(5.3) one adds the summand $\|D u\|_{\Omega}$ to the seminorms of $u$.

Proof. Let $\varphi \in \widehat{S},\left.\varphi\right|_{\partial \Omega}=0$. The error of the modified method satisfies the identity

$$
(\mathcal{L})_{\Omega}(\widehat{u}-v, \varphi)=-\Delta(\mathcal{L})_{\Omega}(u, \varphi)-(\mathcal{L})_{\Omega}(u-\widehat{u}, \varphi),
$$

where $\Delta(\mathcal{L})_{\Omega}(u, \varphi)=\mathcal{L}_{\Omega}(u, \varphi)-(\mathcal{L})_{\Omega}(u, \varphi)$. The estimation of the bilinear form $(\mathcal{L})_{\Omega}(u-\widehat{u}, \varphi)$ is accomplished similar to the same one in the proof of Theorem 5.1 and even simpler.

To bound $\Delta(\mathcal{L})_{\Omega}(u, \varphi)$, we write down the formula

$$
\Delta(\mathcal{L})_{\Omega}(u, \varphi)=\sum_{i, j=1,2}\left(\int_{\Omega^{(i)}}\left(a_{i j}-\left\langle a_{i j}\right\rangle_{i}\right) D_{j} u D_{i} \varphi d x+\int_{\Omega^{(i)}}\left(\left\langle a_{i j}\right\rangle_{i}-\left(a_{i j}\right)_{i}\right) D_{j} u D_{i} \varphi d x\right) .
$$

Since $\int_{\Omega^{(k)}}\left(w-\langle w\rangle_{k}\right)\langle\psi\rangle_{k} d x=0$ for any $w, \psi \in L^{1}(\Omega)$ and $\left\langle D_{k} \varphi\right\rangle_{k}=D_{k} \varphi$, for $k=1,2$, the term $D_{j} u$ can be replaced by $D_{j} u-\left\langle D_{j} u\right\rangle_{i}$ in the first summand of the right-hand side. Therefore, by exploiting the inequalities

$$
\left\|w-\langle w\rangle_{k}\right\|_{L^{p}(\Omega)} \leqslant c|h|\|D w\|_{L^{p}(\Omega)}, \quad\left\|\langle w\rangle_{k}-(w)_{k}\right\|_{L^{\infty}(\Omega)} \leqslant c|h|^{2}\left\|D^{2} w\right\|_{L^{\infty}(\Omega)},
$$

where $1 \leqslant p \leqslant \infty$, the desired estimate appears

$$
\left|\Delta(\mathcal{L})_{\Omega}(u, \varphi)\right| \leqslant c|h|^{2} \sum_{i, j=1,2}\left(\left\|D a_{i j}\right\|_{L^{\infty}(\Omega)}\left\|D^{2} u\right\|_{\Omega}+\left\|D^{2} a_{i j}\right\|_{L^{\infty}(\Omega)}\|D u\|_{\Omega}\right)\|\varphi\|_{H^{(1)}} .
$$

Remark 5.1. Of course, it is possible to include into consideration the pointwise approximation of the coefficients $b_{i}$, $a$, as well but we omit this point.

Let now the coefficients of $L$ satisfy the conditions from Section 2.3 as well. We give the error estimates in terms of data.

Theorem 5.2. The following error estimate holds:

$$
\|\widehat{u}-v\|_{H^{\langle 1\rangle}} \leqslant c|h|^{1+\alpha}\left(\left\|f-L g_{\Omega}\right\|_{V^{\alpha}}+\left\|D^{2} g_{\Omega}\right\|^{(\alpha)}\right), \quad 0 \leqslant \alpha \leqslant 1,
$$

where the condition $g_{\Omega} \in W^{2,2}(\Omega)$ is supposed.

Corollary 5.2. Let $|h| \leqslant \frac{1}{2}$. The following error estimates hold:

$$
\begin{aligned}
& \|\widehat{u}-v\|_{H^{\langle 1\rangle}} \leqslant c|h|^{2}|\log | h\left\|^{1 / 2}\right\| f \|_{S_{1}^{\chi} \mathcal{H}} \quad \text { for } g=0, \\
& \|\widehat{u}-v\|_{H^{\langle 1\rangle}} \leqslant c|h|^{1+\alpha}\left(\|f\|_{V^{\langle\alpha\rangle}}+\left\|g_{\Omega}\right\|^{(1)}+c_{\alpha}\left\|D^{2} g_{\Omega}\right\|_{H^{\alpha, 2}(\Omega)}\right)
\end{aligned}
$$

for $0<\alpha<1, \alpha \neq \frac{1}{2}$,

$$
\begin{aligned}
& \|\widehat{u}-v\|_{H^{\langle 1\rangle}} \leqslant c|h|^{3 / 2}\left(\|f\|_{V^{\langle 1 / 2\rangle}}+\left\|g_{\Omega}\right\|^{(1)}+\left\|D^{2} g_{\Omega}\right\|_{H^{1 / 2,2}(\Omega) \cap V^{\langle 1 / 2\rangle}}\right), \\
& \|\widehat{u}-v\|_{H^{\langle 1\rangle}} \leqslant c|h|^{3 / 2}|\log | h \|\left(\|f\|_{\mathfrak{S} H^{1 / 2 \chi_{k}, 2}(\Omega)}+\left\|g_{\Omega}\right\|^{(1)}+\left\|D^{2} g_{\Omega}\right\|_{H^{1 / 2,2}(\Omega)}\right), \\
& \|\widehat{u}-v\|_{H^{\langle 1\rangle}} \leqslant c|h|^{2}|\log | h \|^{\sigma}\left(\|f\|_{\mathfrak{S} B^{(k)}}+\left\|g_{\Omega}\right\|^{(1)}+\sum_{i=1,2} \sum_{j: j \neq i}\left\|D_{i}^{2} g_{\Omega}\right\|_{B^{(j)}}\right),
\end{aligned}
$$

where $\sigma$ and $B^{(k)}$ are the same as in estimate (3.18). 
Proof. The error estimates in Theorem 5.2 and its Corollary are the counterparts of the error estimates proved in Section 3 for another finite element method (the estimates differ not greatly by the norms of $g_{\Omega}$ ). The validity of these estimates follows from the fact that the specific character of the method was not used in the proofs of the results of Section 3 , but only the validity of Theorem 2.1 was important. It suffices to see that Theorem 5.1 replaces the latter.

In addition, these estimates are valid for the modified method from Corollary 5.1 (but only in estimate (5.11) the summand $\left\|D g_{\Omega}\right\|_{\Omega}$ must be added to the seminorm of $g_{\Omega}$ ).

\section{Appendix}

Proof of Theorem 2.2. 1. First, we prove inequality (2.24). Note that

$$
\|L w\|^{\left(\chi_{k}\right)} \leqslant c_{3}\left(\|w\|_{W^{2,2}(\Omega)}+\left\|D_{k} w\right\|_{W^{2,2}(\Omega)}\right)
$$

by virtue of the conditions on the coefficients of $L$ (the constant $c_{3}$ depends on $N, X_{1}, \ldots, X_{n}$, and $n$ only). In addition, if

$$
u, D_{k} u \in W^{2,2}(\Omega),\left.\quad u\right|_{\partial \Omega}=0
$$

then, for $L_{\ell} u=-D_{\ell}\left(a_{\ell \ell} D_{\ell} u\right)+b_{\ell} D_{\ell} u$, we have $\left.L_{\ell} u\right|_{\Gamma_{k}}=0$ for all $\ell, k$ such that $\ell \neq k$. Since $L u=\sum_{i=1}^{n} L_{i} u+a u$, we find $(L u)_{\Gamma_{k}}^{0}=\left.L_{k} u\right|_{\partial \Omega}$. By applying the embedding (trace) theorem from $W^{1,2}(\Omega)$ in $W^{1 / 2,2}(\partial \Omega)$, we obtain

$$
\left\|(L u)_{\Gamma_{k}}^{0}\right\|_{W^{1 / 2,2}(\partial \Omega)} \leqslant c\left\|L_{k} u\right\|_{W^{1,2}(\Omega)} \leqslant c^{\prime}\left\|D_{k} u\right\|_{W^{2,2}(\Omega)} .
$$

Thus, inequality (2.24) for $f=L u$ is proved.

2. The proof of inequality (2.23) is substantially more difficult. First, we establish this inequality for $L=\widetilde{L}$, where $\widetilde{L} w=-\sum_{i=1}^{n} D_{i}\left(a_{i i} D_{i} w-a_{i} w\right)+a_{0} w$ with $a_{k}=D_{k} a_{k k}, 1 \leqslant$ $k \leqslant n$, and sufficiently large constant $a_{0}$.

For $w \in C^{4}(\bar{\Omega})$ such that $\left.w\right|_{\partial \Omega}=0,\left.D_{k}^{2} w\right|_{\Gamma_{k}}=0$, the following formula holds:

$$
\begin{aligned}
\int_{\Omega}\left[D _ { k } D _ { \ell } \left(a_{\ell \ell} D_{\ell} w\right.\right. & \left.\left.-a_{\ell} w\right)\right] D_{k} D_{m}^{2} w d x \\
& =\int_{\Omega}\left[D_{k} D_{m}\left(a_{\ell \ell} D_{\ell} w-a_{\ell} w\right)\right] D_{k} D_{m} D_{\ell} w d x, \quad \forall \ell, m .
\end{aligned}
$$

For $\ell \neq m$ (only this case is nontrivial), this formula is derived by transferring first $D_{\ell}$ and next $D_{m}$ from one cofactor to another; the substitutions equal zero according to the choice of $w$ and $a_{\ell}$.

We temporally suppose that the function $u$ satisfies conditions (6.1) and $\left.D_{k}^{2} u\right|_{\Gamma_{k}}=0$. Formula (6.2) is valid for $w=u$ as well; to verify this, it suffices to extend $u$ (oddly with respect to the variables $x_{k}$ sequentially for $\left.k=1, \ldots, n\right)$, to mollify the result using a smooth, and even with respect to all variables, kernel, and to pass to the limit as the radius of the mollifier tends to 0 . By exploiting this formula to transform the integral 
$\int_{\Omega} D_{k} \widetilde{L} u \cdot D_{k}(-\Delta) u d x$ with $\Delta$ being the Laplace operator, and accomplishing actions similar to those given in Section 3.7 in [11], we obtain the inequality

$$
\frac{\nu}{2} \sum_{i, j=1}^{n}\left\|D_{k} D_{i} D_{j} u\right\|_{\Omega}^{2} \leqslant\left\|D_{k} \widetilde{L} u\right\|_{\Omega}^{2}+c\|u\|_{H^{\langle 2\rangle}}^{2}
$$

By applying also inequality (2.5) in the case of $L=\widetilde{L}$ and $g=0$, we get

$$
\|u\|_{H^{\langle 2\rangle}}+\left\|D_{k} u\right\|_{W^{2,2}(\Omega)} \leqslant c\left(\|\widetilde{L} u\|_{\Omega}+\left\|D_{k} \widetilde{L} u\right\|_{\Omega}\right) .
$$

The constants $c$ in the latter inequalities depend on $\nu, N, X_{1}, \ldots, X_{n}, n$, and $a_{0}$ only.

For $-L=\Delta$ being the Laplace operator, by exploiting the Fourier expansion with respect to sines, one can verify that the suggestion $f \in W_{0}^{\chi_{k}, 2}(\Omega)$ implies the validity of conditions (6.1) and the property $\left.D_{k}^{2} u\right|_{\Gamma_{k}}=0$. To establish the same for $L=\widetilde{L}$, it suffices to apply the continuation, with respect to a parameter, method, see Section 3.9 in [11] by basing on a priori estimate (6.3) and the results of Item 1 of the proof. Thus, inequality (2.23) is valid for $L=\widetilde{L}$ and $f \in W_{0}^{\chi_{k}, 2}(\Omega)$.

For $f \in W^{\chi_{k}, 2}(\Omega)$, the additional condition $\left.f\right|_{\Gamma_{k}}=0$ can be removed by exploiting the extension theorem (more precisely, the converse trace theorem) from $W^{1 / 2,2}(\partial \Omega)$ to $W^{1,2}(\Omega)$. According to this theorem, there exists a function $F$ with the properties

$$
\|F\|_{W^{1,2}(\Omega)} \leqslant c\left\|(f)_{\Gamma_{k}}^{0}\right\|_{W^{1 / 2,2}(\partial \Omega)},\left.\quad F\right|_{\partial \Omega}=(f)_{\Gamma_{k}}^{0} .
$$

Then $f=(f-F)+F$ with $\left.(f-F)\right|_{\Gamma_{k}}=0$ and $\left.F\right|_{\Gamma_{\ell}}=0$ for $\ell \neq k$. It remains to note that inequality (2.23) has been proved above though for $\left.f\right|_{\Gamma_{k}}=0$, but for all $k$.

Finally, the case of the general operator $L$ is reduced to the case of $L=\widetilde{L}$ by applying the formulas

$$
\widetilde{L} u=L u+\widetilde{f}=f+\widetilde{f}, \quad \tilde{f}=\sum_{i=1}^{n}\left(a_{i}-b_{i}\right) D_{i} u+\left(\operatorname{diva}+a_{0}-a\right) u
$$

Really, taking into account inequality (2.5), the properties

$$
\left(\left(a_{\ell}-b_{\ell}\right) D_{\ell} u\right)_{\Gamma_{k}}^{0}= \begin{cases}\left.\left(\left(a_{\ell}-b_{\ell}\right) D_{\ell} u\right)\right|_{\partial \Omega} & \text { for } k=\ell \\ 0 & \text { for } k \neq \ell\end{cases}
$$

and the embedding (trace) theorem from $W^{1,2}(\Omega)$ in $W^{1 / 2,2}(\partial \Omega)$, we get

$$
\|\widetilde{f}\|^{\left(\chi_{k}\right)}+\left\|(\widetilde{f})_{\Gamma_{k}}^{0}\right\|_{W^{1 / 2,2}(\partial \Omega)} \leqslant c\|u\|_{H^{\langle 2\rangle}} \leqslant c^{\prime}\|f\|_{\Omega}
$$

that completes the proof.

Remark 6.1. Let $b_{1}=\cdots=b_{n}=a=0, g=0$, and $n \geqslant 2$. For any $\varepsilon>0$, one can find the constant coefficients $a_{i j}$ (satisfying the ellipticity condition (2.3)) and an arbitrarily smooth, in $\bar{\Omega}$, function $f$ with $\left.f\right|_{\partial \Omega}=0$ such that the solution to the problem $(2.1),(2.2)$ does not belong to $W^{2,2+\varepsilon}(\Omega)$ and, moreover, to $W^{3,2}(\Omega)$. Therefore, the condition $a_{i j}=0$ for all $i \neq j$ cannot be removed in Theorem 2.2 (though this can be weakened). 


\section{References}

[1] T. I. Amanov, Spaces of Functions with a Dominating Mixed Derivative, Nauka, Alma-Ata, 1976 (in Russian).

[2] N. S. Bakhvalov, B. I. Berezin, and Yu. L. Bessonov, Interpolation theorems for operators subordinated to several derivatives, Dokl. AN SSSR, 256 (1981), No. 6, pp. 1296-1300 (in Russian).

[3] B. I. Berezin, Rate of convergence estimate for a difference scheme for the Poisson equation with the piecewise smooth right-hand side, Vestn. MGU, Ser. 15, Comput. Math. Cybern., 3 (1979), No. 2, pp. 26-31 (in Russian).

[4] J. Bergh and J. Löfström, Interpolation Spaces: an Introduction, Springer-Verlag, Berlin, 1976.

[5] O. V. Besov, V. P. Il'in, and S. M. Nikolskii, Integral Representations of Functions and Embedding Theorems, Halsted Press Books, 1979.

[6] P. Ciarlet, The Finite Elemant Method for Elliptic Problems, North-Holland, Amsterdam, 1978.

[7] R. Z. Dautov, A. V. Lapin, and A. D. Lyashko, Some mesh schemes for quasilinear elliptic equations, Comp. Maths. Math. Phys., 20 (1980), No. 2, pp. 62-78.

[8] M. Křižek and P. Neitaanmäki, On superconvergence technique, Acta Appl. Math., 9 (1987), No. 3, pp. 175-198.

[9] M. Křižek and P. Neitaanmäki, Bibliography on superconvergence, in: Finite Element Methods: Superconvergence, Post-Processing, and A Posteriori Estimates (M. Křižek et al., eds), Marcel Dekker, N.Y., 1997, pp. 315-348.

[10] N. N. Kuznetsov, Weak solutions to quasilinear equations and numerical methods of their calculation, Banach Center Publ., 3 (1978), pp. 9-39 (in Russian).

[11] O. A. Ladyzhenskaya and N. N. Ural'ceva, Linear and Quasilinear Elliptic Equations, 2nd ed., Nauka, Moscow, 1973 (in Russian); English transl. of 1st ed., Academic Press, 1968.

[12] R. D. Lazarov, V. L. Makarov, Convergence of the mesh method and method of lines for multidimensional problems of mathematical physics in the classes of generalized solutions, Dokl. AN SSSR, 259 (1981), No. 2, pp. 282-286 (in Russian).

[13] S. M. Nikolskii, On one property of classes $H_{p}^{r}$, Ann. Univ. Sc. Budapest, Sectio Math., III-IV (1960/1961), pp. 205-216.

[14] S. M. Nikolskii, Approximation of Functions of Several Variables and Embedding Theorems, Nauka, Moscow, 1969 (in Russian); English transl., Springer-Verlag, 1974.

[15] L. A. Oganesyan and L. A. Rukhovets, Variational-difference Methods for Solving Elliptic Equations, Armenian Academy of Sciences, Erevan, 1979 (in Russian).

[16] A. A. Samarskii, R. D. Lazarov, and V. L. Makarov, Difference Schemes for Differential Equations with Generalized Solutions, V'ysshaya Shkola, Moscow, 1987 (in Russian).

[17] H. Triebel, Interpolation Theory. Function spaces. Differential Operators, VEB Deitscher Verlag, Berlin, 1978.

[18] A. I. Vol'pert and S. I. Khudyaev, Analysis in the Classes of Discontinuous Functions, and the Equations of Mathematical Physics, Nauka, Moscow, 1975 (in Russian).

[19] L. B. Wahlbin, Superconvergence in Galerkin Finite Element Methods, Lecture Notes in Math., 1605, Springer-Verlag, Berlin, 1995.

[20] A. A. Zlotnik, Projection-difference scheme for a one-dimensional parabolic equation with a nonsmooth solution, Moscow Univ. Comp. Math. Cybern., 1 (1977), No. 1, pp. 62-66.

[21] A. A. Zlotnik, A projection-difference scheme for the vibrating-string equation, Soviet. Math. Dokl., 20 (1979), No. 2, pp. 290-293.

[22] A. A. Zlotnik, Projection-difference schemes for nonstationary problems with nonsmooth data, Ph. D. thesis, Department of Cybernetics and Computational Mathematics, Moscow State Univ., Moscow, 1979, 146 pp. (in Russian). 
[23] A. A. Zlotnik, On the rate of convergence in $W_{2, h}^{1}$ of the variational-difference method for elliptic equations, Soviet. Math. Dokl., 28 (1983), No. 1, pp. 143-148.

[24] A. A. Zlotnik, Some finite-element and finite-difference methods for solving mathematical physics problems with nonsmooth data in n-dimensional cube. Part I, Sov. J. Numer. Anal. Math. Modell., 6 (1991), No. 5, pp. 421-451.

[25] A. A. Zlotnik, Toward the theory of projection-difference methods for mathematical physics problems with nonsmooth data, D. Sc. thesis, Department of Mathematics, Moscow Pedagogical State Univ., Moscow, 1992, 366 pp. (in Russian).

[26] A. A. Zlotnik, Convergence rate estimates of finite-element methods for second order hyperbolic equations, in: Numerical Methods and Applications (G. I. Marchuk, ed.), CRC Press, Boca Raton, 1994, pp. $155-220$. 